\title{
On the consequences of a non-equilibrium ionisation balance for compact flare emission and dynamics ${ }^{\star}$
}

\author{
S. J. Bradshaw ${ }^{1}$, G. Del Zanna ${ }^{2}$, and H. E. Mason ${ }^{2}$ \\ 1 Blackett Laboratory, Imperial College London, Prince Consort Road, London SW7 2BZ, UK \\ e-mail: s.bradshaw@imperial .ac.uk \\ 2 Department of Applied Mathematics and Theoretical Physics, Centre for Mathematical Sciences, University of Cambridge, \\ Wilberforce Road, Cambridge CB3 0WA, UK
}

Received 25 March 2004 / Accepted 1 June 2004

\begin{abstract}
We carry out a hydrodynamic simulation of a compact flare and find significant non-equilibrium distributions for the ionisation balance during the impulsive and gradual phases, which can strongly alter the radiative emission. This has major implications for attempts to derive the theoretical intensities of emission lines used for spectroscopic diagnostic analyses of the plasma properties.

During the impulsive phase we find that the emissivities of He I, He II and C IV in the transition region can be strongly enhanced above their expected equilibrium values, followed by a significant reduction which increases the amount of chromospheric plasma ablated into the corona. Furthermore, during the flare heating the overall charge state of the coronal ions can be significantly lower than is suggested by an equilibrium ionisation balance and, therefore, line ratio measurements will yield plasma temperatures that are much greater than the formation temperature of the emitting ion.

During the gradual phase the emissivity at transition region temperatures remains suppressed, compared with its equilibrium value, with correspondingly reduced downflow velocities and increased radiative cooling time-scales.

Finally, we synthesise the emission as it would be detected by TRACE in its $171 \AA$ and $195 \AA$ wavelength bands, and find that the filter ratio technique can give reasonably good estimates of the plasma temperature in quiescence, though when the populations of Fe VIII, Fe IX, Fe X and Fe XII exhibit departures from equilibrium the temperatures derived from filter ratio measurements become unreliable.
\end{abstract}

Key words. Sun: flares - hydrodynamics - atomic processes - Sun: UV radiation

\section{Introduction}

The current paper is the final of a series of three in which the consequences of a non-equilibrium ionisation balance for observations and theoretical models of the solar atmosphere are investigated. In Bradshaw \& Mason (2003a, hereafter BM1) we simulated a loop cooling from coronal to transition region temperatures and in Bradshaw \& Mason (2003b, hereafter BM2) we simulated a small-scale heating event with properties characteristic of a nanoflare. We used a new hydrodynamic code (HYDRAD), which is designed to couple the time-dependent ionisation balance equations to the hydrodynamic equations in a self-consistent manner. We found that non-equilibrium effects can become important in both scenarios, which are commonly assumed to occur in the solar atmosphere, and studied the consequences of a non-equilibrium ionisation balance for the radiative emission and the dynamics of the plasma. In the current paper we simulate a compact flare of moderate energy and follow the evolution of the plasma through the impulsive and

* Figures 4-24 are only available in electronic form at http://www . edpsciences.org gradual phases. The term compact flare describes a strong heating event that takes place in a closed magnetic loop and the field remains essentially unchanged for its duration.

Among the first self-consistent compact flare models were those of MacNeice et al. (1984), Nagai \& Emslie (1984) and Fisher et al. (1985). Their computational codes modelled a chromosphere and a corona coupled via thermal conduction across a thin transition region, and followed the hydrodynamic response of an initially hydrostatic atmosphere to thick-target heating of the chromosphere. The simulations of MacNeice et al. (1984) and Nagai \& Emslie (1984) under-resolved the transition region, whereas the computational code used by Fisher et al. (1985) included an adaptive regridding scheme (McClymont \& Canfield 1983) to ensure sufficient resolution everywhere along the loop.

Several thermal heating models of compact flares have also been published. Nagai (1980), Nagai et al. (1983), Cheng et al. (1983), Pallavicini et al. (1983) and MacNeice (1986) have all investigated the effect of depositing the flare energy at the loop apex. The transition region was under-resolved by all of these authors with the exception of MacNeice (1986), who 
incorporated an adaptive regridding scheme into the computational code.

The constraints imposed on the allowable time-step by the spatial-scale of a properly resolved transition region mean that it can be prohibitively expensive (in terms of real-time and the monetary cost of computing time) to carry out a complete hydrodynamic simulation of a flare from the initial input of heat, through the downward propagation of the transition region and the upward evaporation of chromospheric material, to the eventual cooling and condensation of the evaporated material back onto the chromosphere (MacNeice 1986).

A major consequence of insufficient resolution in the transition region is the under-estimation of the conducted heat flux, which is due to the artificial limitation of the temperature gradient to a resolution dependent maximum value. This leaves energy bottled up in the corona and may result in the over-estimation of coronal temperatures, as well as the underestimation of chromospheric ablation velocities and coronal densities, in numerical simulations.

The physical treatment of the conducted heat flux in the transition region was considered in detail by MacNeice et al. (1984), Nagai \& Emslie (1984) and Fisher et al. (1985). The classical treatment of Spitzer (1962), a common feature of both hydrostatic and hydrodynamic models to-date, allows the conducted heat flux to increase without limit as the temperature gradient steepens. Eventually the conducted flux calculated in this way will become unphysical because its upper limit is prescribed by the free-streaming value, which occurs when all of the electrons move at the thermal speed in the same direction. MacNeice et al. (1984) and Nagai \& Emslie (1984) used the lesser of the classical (Spitzer) heat flux and the saturated flux as the actual conducted heat flux. However, there are associated problems because the derivative of the conducted heat flux, which appears in the energy balance, is not treated selfconsistently. Fisher et al. (1985) adopted a scheme whereby there is a smooth transition between the values for the classical and saturated regimes. Karpen \& Devore (1987) included the effects of non-local thermal transport in their simulations of solar flares.

Antiochos \& Krall (1979), Doschek et al. (1982) and Gan \& Fang (1990) have studied the gradual or cooling phase of a flare, while circumventing consideration of the impulsive phase, by simulating the cooling of a flare loop in an initial hydrostatic equilibrium. Antiochos \& Krall (1979) and Doschek et al. (1982) took as their initial conditions an apex temperature of $17.6 \times 10^{6} \mathrm{~K}$, a density of $5.6 \times 10^{11} \mathrm{~cm}^{-3}$ and a total loop length of $5 \times 10^{8} \mathrm{~cm}$. Gan \& Fang (1990) used an initial apex temperature of $2 \times 10^{7} \mathrm{~K}$, a density of $10^{10} \mathrm{~cm}^{-3}$ and a total loop length of $6 \times 10^{9} \mathrm{~cm}$.

To simulate the onset of the gradual phase Antiochos \& Krall (1979) and Doschek et al. (1982) simply switched off the heating term in the energy balance, whereas Gan \& Fang (1990) added a flare heating component to the energy balance and linearly decreased it to zero during the first $10 \mathrm{~s}$ of their simulation. During the earliest part of the gradual phase thermal conduction continued to drive chromospheric evaporation and all of the models predicted similar velocities for the ablated material. This is consistent with observations, which have shown that the peak in emission measure is reached after the peak in temperature.

Cargill et al. (1995) derived analytical formulae relating the total cooling time to the loop half-length and the pressure or density (in the corona) when cooling commences. They assumed that cooling of the flare plasma is first dominated by conduction and then by radiation, giving formulae for the cooling time-scales of both phases:

$\tau_{\mathrm{c}}=4 \times 10^{-10} \frac{n L^{2}}{T^{\frac{5}{2}}}$,
$\tau_{\mathrm{r}}=\frac{3 k_{\mathrm{B}}}{n \chi T^{\alpha-1}}$.

The results of Gan \& Fang (1990) showed a much longer cooling time (about $20 \mathrm{~min}$ ) than those of Antiochos \& Krall (1979) and Doschek et al. (1982) (about 2 min). Cargill et al. (1995) also found good agreement between the former results and the predicted cooling times given by their analytical formulae. The longer cooling times agree more closely with observations of cooling compact flare loops.

There are a number of possibilites that may explain the different cooling time-scales obtained by Antiochos \& Krall (1979) and Doschek et al. (1982), and Gan \& Fang (1990). The earlier two papers adopted very different initial conditions to the latter paper, which could influence the nature of the plasma cooling. For example, from Eq. (1) above, it would appear that Antiochos \& Krall (1979) and Doschek et al. (1982) should have obtained the longest cooling time-scales because of the higher density used in their initial conditions, however, this is clearly not the case and Eq. (2) suggests that in fact radiative cooling dominates in their simulations. This is further supported by the fact that Gan \& Fang (1990) used a loop length an order of magnitude greater than the other two papers and Eq. (1) shows that $\tau_{\mathrm{c}} \propto L^{2}$. A reasonably self-consistent picture then emerges from these considerations, where conductive cooling quickly gives way to an extremely rapid radiative cooling in the case of Antiochos \& Krall (1979) and Doschek et al. (1982), whereas in the case of Gan \& Fang (1990) both the conductive and radiative cooling time-scales are greater.

The results of Antiochos \& Krall (1979) and Doschek et al. (1982) may have been influenced by substantially overestimating the chromospheric radiative energy loss and underresolving the transition region. Gan \& Fang (1990) carried out a careful investigation of the chromospheric radiative losses in order to choose an appropriate radiative loss function for low temperature and high density plasma, and they also employed a higher resolution computational grid. If radiation is over-estimated in the transition region and the chromosphere then more heat via thermal conduction from the corona is required to balance the energy loss. If the transition region is under-resolved then it may not be able to handle the necessary heat flux and this results in its rapid ascent along the loop (as in Doschek et al. 1982) because the plasma beneath it quickly cools to chromospheric temperatures. In Gan \& Fang (1990) the transition region continued to move downward during the early part of the gradual phase and then remained stationary for a long while, before beginning to ascend at the latter stages of the gradual phase. 
Evidently it is necessary to carry out hydrodynamic simulations for the entire duration of a flare in order to perform a self-consistent study. The nature of cooling clearly depends upon the properties of the flare plasma, which in turn depend upon the details of how the plasma was heated.

In light of the work reviewed above we decided to apply HYDRAD to the problem of self-consistently modelling a solar flare throughout every phase of its evolution and to investigate the effects of non-equilibrium processes on the ion populations and their associated radiative emission. Thus, quantitative predictions can be made regarding the consequences of these nonequilibrium processes for theoretical and observational studies of flaring plasma.

The paper is structured as follows: Sect. 2 gives details of our model; Sect. 3 describes the results from the impulsive phase of the flare; Sect. 4 describes the results from the gradual phase of the flare; Sect. 5 discusses the observational consequences by synthesising the emission detectable by the TRACE $171 \AA$ and $195 \AA$ wavelength bands; and Sect. 6 gives an overall summary of the results and conclusions.

\section{Simulating a compact flare with HYDRAD}

The nature of the flare heating mechanism chosen for the current work is a thermal perturbation at the apex of the loop, rather than a mechanism such as beam heating in the chromosphere. This choice was partly motivated by the conclusions of others that a thermal heating mechanism produces results more consistent with observations (Peres et al. 1987; Betta et al. 2001) and partly by observations themselves where evidence for heating via magnetic reconnection events taking place near the loop apex has been found (Masuda et al. 1995; Shibata et al. 1995). However, there is also evidence that some flares are driven by magnetic reconnection with newly emerging flux near loop footpoints (Fludra et al. 1995) and consequently the hydrodynamic simulations performed here are applicable only to compact flares driven by an assumed thermal input of energy in the apex region of the loop.

The thermal perturbation applied to the initial loop atmosphere at $t>0$ is of the form:

$E_{\mathrm{H}}(s, t)=E_{\mathrm{Hbg}}+E_{\mathrm{H} 0} \exp \left(-\frac{\left|s-s_{0}\right|}{s_{\mathrm{H}}}\right) \sin \left(\pi \frac{t-t_{0}}{\tau_{\mathrm{H}}}\right)$.

Here $E_{\mathrm{Hbg}}$ and $E_{\mathrm{H} 0}$ are the background heat input required to maintain the initial atmosphere and the maximum transient heat input. The exponential term describes the spatial distribution of heat, where $s_{0}$ and $s_{\mathrm{H}}$ are the location of maximum heating and the heating scale length (or e-folding length). The sinusoidal term describes the transient behaviour of the heating pulse, where $t_{0}$ and $\tau_{\mathrm{H}}$ are the onset time and the period (ie. the time taken for it to rise, reach its maximum and decay).

The following values were adopted for the parameters:

$E_{\mathrm{BG}}=2.4 \times 10^{-4} \mathrm{erg} \mathrm{cm}^{-3} \mathrm{~s}^{-1}, E_{\mathrm{H} 0}=0.1 \mathrm{erg} \mathrm{cm}^{-3} \mathrm{~s}^{-1}, s_{0}=$ $4 \times 10^{9} \mathrm{~cm}, s_{\mathrm{H}}=5 \times 10^{8} \mathrm{~cm}, t_{0}=0 \mathrm{~s}, \tau_{\mathrm{H}}=300 \mathrm{~s}$,

which describe a heating pulse delivered symmetrically about the loop apex with a maximum volumetric heat input of $0.1 \mathrm{erg} \mathrm{cm}^{-3} \mathrm{~s}^{-1}$, a spatial-scale of $5 \times 10^{8} \mathrm{~cm}$ and a period of $300 \mathrm{~s}$. Only one pulse is delivered to the loop in each simulation and so only constant background heating remains after $300 \mathrm{~s}$. The loop eventually returns to its initial hydrostatic equilibrium. The amount of heat delivered to the loop by the pulse is about $2 \times 10^{10} \mathrm{erg} \mathrm{cm}^{-2}$, which is toward the low-energy regime of typical flares, though the plasma still reaches temperatures characteristic of soft X-ray emission, and velocities of $200 \mathrm{~km} \mathrm{~s}^{-1}$.

A new initial loop atmosphere was derived with extended chromospheric regions of $5 \times 10^{8} \mathrm{~cm}$. The extended chromospheres are necessary because the substantially increased conducted heat flux means that the transition region can be expected to propagate further than the $1.5 \times 10^{8} \mathrm{~cm}$ depth of the chromospheres in the initial atmosphere used in BM1 and BM2. Consequently, a deeper chromosphere is required to avoid the numerical influence of boundary effects such as wave reflections and the loss of mass and energy from the loop.

The properties of the initial atmosphere are similar to the average loop properties found by Aschwanden \& Alexander (2001), Table 3, for their study of the Bastille Day flare, and also used by Tsiklauri et al. (2004) who carry out a hydrodynamical simulation of this event. In summary: the length of the loop used in the present study is $8 \times 10^{9} \mathrm{~cm}$ (somewhat longer than the average length of $5.5 \times 10^{9} \mathrm{~cm}$ reported by Aschwanden \& Alexander 2001); the coronal part of the loop has a temperature of $1.6 \times 10^{6} \mathrm{~K}$ at its apex; and the electron number density is approximately $4.7 \times 10^{8} \mathrm{~cm}^{-3}$. Both the temperature and the density are slightly greater than the initial values used by Tsiklauri et al. (2004), though not significantly so. The duration of the heating event in the present study (300 s) is also of the same order as the longer time-scales in the range chosen by Tsiklauri et al. (2004). However, it is not the intention of the authors to attempt a simulation of the individual loops comprising the Bastille Day event in the present study.

The response of the plasma to the flare heating was calculated by solving the one-dimensional, conservative form of the hydrodynamic equations in curvilinear coordinates (taken along the loop in the direction of the magnetic field):

$$
\begin{aligned}
& \frac{\partial \rho}{\partial t}+\frac{\partial}{\partial s}(\rho v)=0, \\
& \frac{\partial}{\partial t}(\rho v)+\frac{\partial}{\partial s}\left(\rho v^{2}\right)=\rho g_{\|}-\frac{\partial P}{\partial s},
\end{aligned}
$$

$$
\begin{aligned}
\frac{\partial E}{\partial t}+\frac{\partial}{\partial s}[(E+P) v]= & \rho v g_{\|}+\frac{\partial}{\partial s}\left(\kappa T^{5 / 2} \frac{\partial T}{\partial s}\right) \\
& +E_{\mathrm{H}}(s, t)-E_{\mathrm{R}}(s, t),
\end{aligned}
$$

$E=\frac{1}{2} \rho v^{2}+\frac{3}{2} k_{\mathrm{B}} n T$,

$P=2 k_{\mathrm{B}} n T$.

Here $\rho, v, P$ and $T$ are the mass density, bulk velocity, total pressure and temperature, respectively; $g_{\|}$is the gravitational acceleration parallel to the magnetic field; $k_{\mathrm{B}}$ is the Boltzman constant; $\kappa$ is the Spitzer coefficient of thermal conductivity and has a value of $9.2 \times 10^{-7} \mathrm{erg} \mathrm{cm}^{-1} \mathrm{~s}^{-1} \mathrm{~K}^{-\frac{7}{2}} ; E_{\mathrm{H}}$ is the volumetric 
heating rate; and $E_{\mathrm{R}}$ is the energy loss rate due to radiation. Note that $E_{\mathrm{R}}$ is a function of $s$ and $t$ and accounts for departures from ionisation equilibrium of the population of emitting ions.

Details of the boundary conditions, the numerical methods employed, and the results of numerical tests designed to assess the stability and the accuracy of the hydrodynamic code are given in BM1 and BM2.

As in BM1 and BM2, two simulations were performed: one employing a straightforward equilibrium treatment of the ion populations (simulation $\mathrm{A}$ ) and the associated radiative emission; and the other employing a full non-equilibrium treatment (simulation B). The equilibrium ionisation balances were calculated using the same ionisation and recombination rates (Mazzotta et al. 1998) as those used to solve the system of time-dependent ionisation balance equations for each element and the element abundances were assumed to be coronal. The individual ion emissivities were calculated using the CHIANTI atomic database (Dere et al. 1997; Young et al. 2003).

\section{The impulsive phase}

\subsection{The dynamical evolution of the plasma}

The impulsive phase of the flare for the purposes of the current work is defined as the period during which the plasma reaches its maximum temperature and the ablated chromospheric material reaches its maximum velocity. Thereafter, the flare plasma begins to cool and enters its gradual phase. Figure 1 shows the heating profile which decays exponentially in space either side of the maximum at the loop apex and obeys a sinusoidal variation in time. The rate of energy input rises from zero, reaches its peak at $150 \mathrm{~s}$ and returns to zero at $300 \mathrm{~s}$. Figure 1 also shows time series of the profiles for a number of physical properties of the plasma between $0 \mathrm{~s}$ and $210 \mathrm{~s}$. Figure 2 shows an enlarged view of the same profiles in the left-hand footpoint region of the loop. There are no discernible differences in the profiles of these properties between simulations $\mathrm{A}$ and $\mathrm{B}$, during the impulsive phase of the flare, because the energy balance in the coronal region of the loop is dominated by heating, thermal conduction and, toward the end of the impulsive phase, convection. Though the dynamical evolution of the loop plasma is almost identical in simulations $\mathrm{A}$ and $\mathrm{B}$, there remain consequences for the overall radiative emission and the diagnostic line intensities, which will affect the results and conclusions drawn from observations and are discussed in the following sections.

After $60 \mathrm{~s}$ of evolution the temperature, pressure and the total energy in the coronal region of the loop have dramatically increased, by a factor of 3 at the apex, and downflows of around $2 \times 10^{6} \mathrm{~cm} \mathrm{~s}^{-1}$ have developed. The sound speed has also increased in response to the rising temperature and is never exceeded by the plasma velocity in the coronal region. The density remains almost constant since the downflows are not substantial enough to carry a significant amount of material away. The conducted heat flux has also increased, by more than an order of magnitude at its peak, in response to the
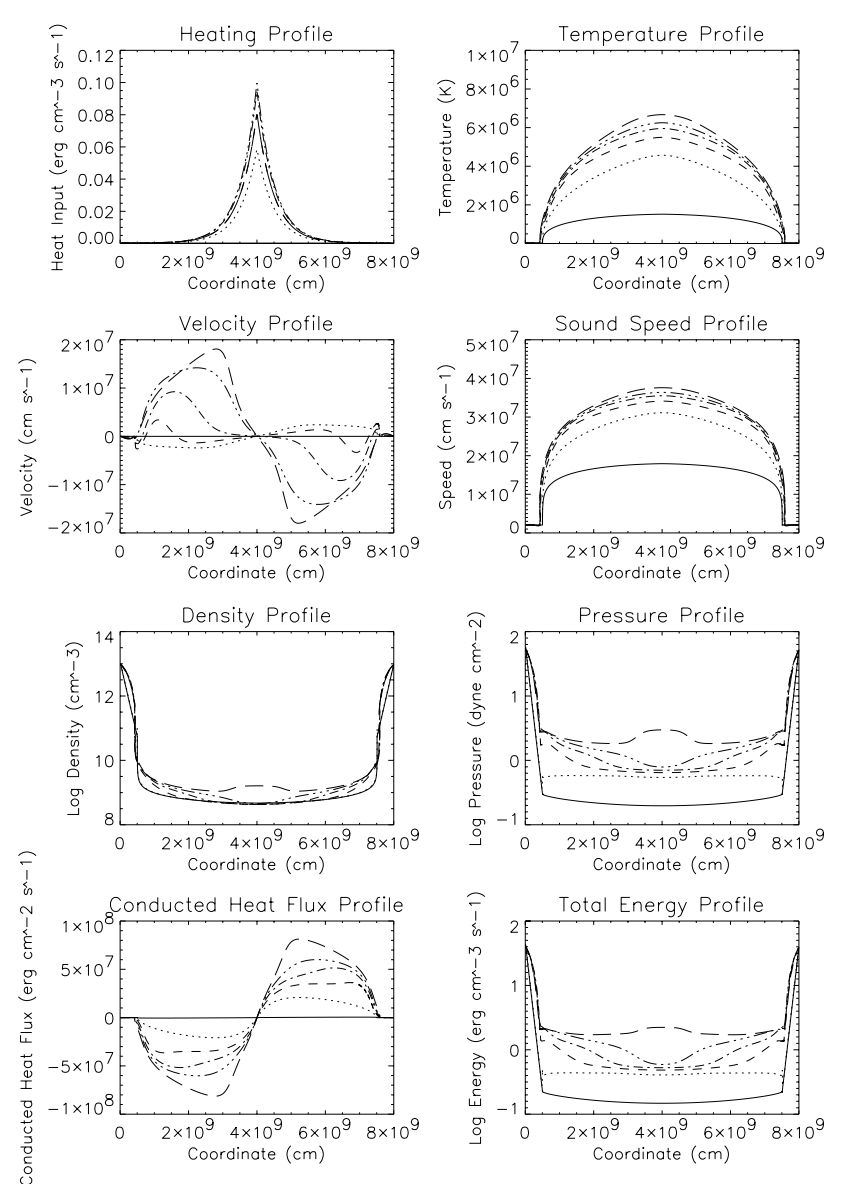

Fig. 1. Time series of the heating, temperature, velocity, sound speed, density, pressure, conducted heat flux and total energy along the length of the loop at: $0 \mathrm{~s}$ (solid), $60 \mathrm{~s}$ (dotted), $90 \mathrm{~s}$ (dashed), $120 \mathrm{~s}$ (dashdot), $150 \mathrm{~s}$ (dash-dot-dot-dot) and $210 \mathrm{~s}$ (long-dashed). The profiles are taken from simulation $\mathrm{A}$.

increasing coronal temperature and the steepening coronal temperature gradient.

Figure 2 shows that after $90 \mathrm{~s}$ the conduction front associated with the coronal heating event has propagated into the chromosphere to a depth of about $500 \mathrm{~km}$ and increased the temperature of the plasma to values on the order of $10^{5} \mathrm{~K}$. The density and pressure profiles show that a large amount of chromospheric material has been ablated into the corona due to the inability of the chromosphere to efficiently radiate the excess energy. Material has also been pushed further down into the chromosphere by the oppositely directed flows that arise in response to the ablation.

The temperature, the conducted heat flux and the total energy continue to increase between $90 \mathrm{~s}$ and $210 \mathrm{~s}$ in the coronal region of the loop as the ablation accelerates and fills it with material. The temperature in the apex region of the loop reaches $7 \times 10^{6} \mathrm{~K}$; the conducted heat flux peaks at $8 \times 10^{7} \mathrm{erg} \mathrm{cm}^{-2} \mathrm{~s}^{-1}$ compared with a peak value at $t=0 \mathrm{~s}$ of $5 \times 10^{5} \mathrm{erg} \mathrm{cm}^{-2} \mathrm{~s}^{-1}$; and the pressure increases by a factor $\geq 10$ in most of the coronal region (as does the total energy). The upflows reach velocities close to $2 \times 10^{7} \mathrm{~cm} \mathrm{~s}^{-1}$ and substantially increase the density of the coronal plasma. The upflowing material in each half of the loop eventually collides, producing a localised 

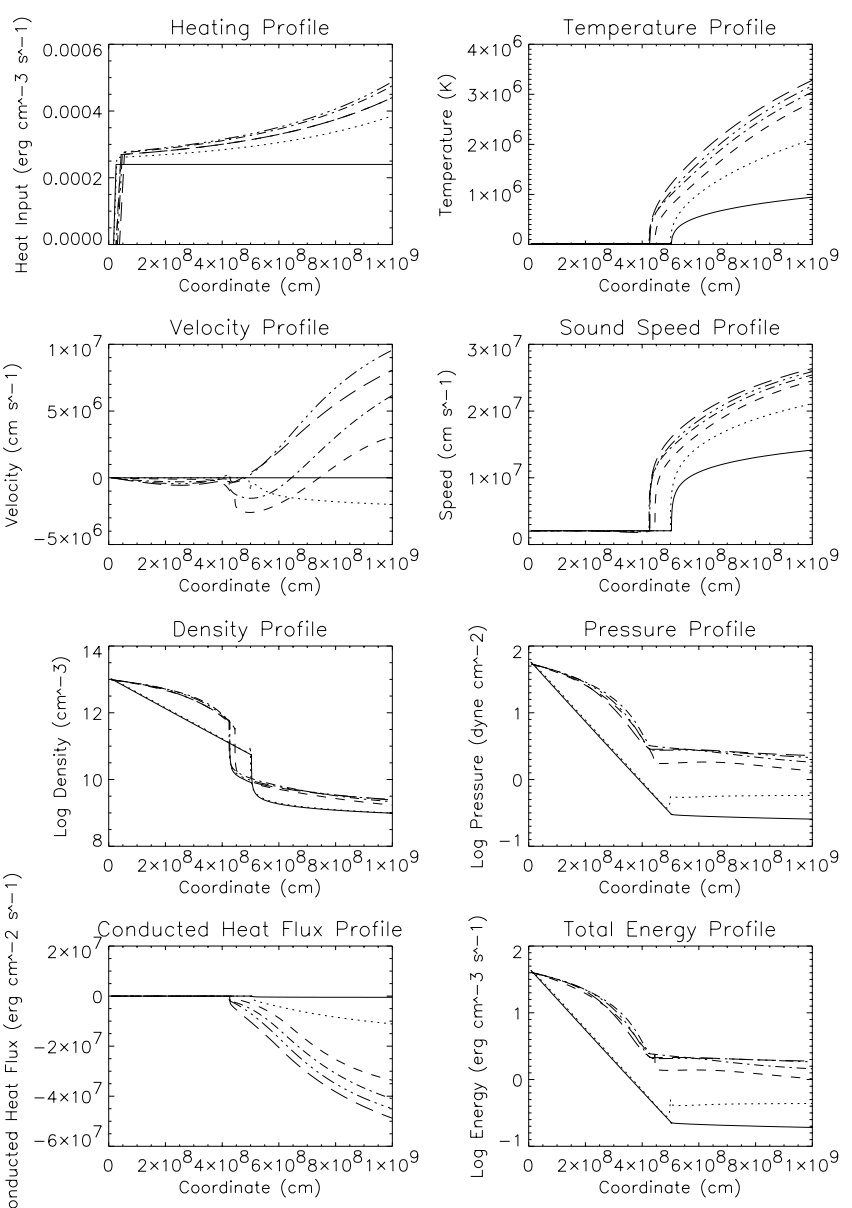

Fig. 2. Time series of the heating, temperature, velocity, sound speed, density, pressure, conducted heat flux and total energy in the left-hand footpoint region of the loop at: $0 \mathrm{~s}$ (solid), $60 \mathrm{~s}$ (dotted), $90 \mathrm{~s}$ (dashed), $120 \mathrm{~s}$ (dash-dot), $150 \mathrm{~s}$ (dash-dot-dot-dot) and $210 \mathrm{~s}$ (long-dashed). The profiles are taken from simulation A.

enhancement in the density, pressure and total energy in the apex region. The density enhancement is a factor of 3 greater than the initial apex value. The pressure and total energy enhancement is a factor of 16 . The observational consequences would be a localised enhancement in the measured intensity of radiative emission.

In the footpoint region the conduction front continues to propagate into the chromosphere, reaching a depth of $750 \mathrm{~km}$ at $210 \mathrm{~s}$, as the temperature of the initially chromospheric material rises to a maximum value of $1.5 \times 10^{6} \mathrm{~K}$. The conducted heat flux carried by the transition region increases to values on the order of $10^{6}$ to $10^{7} \mathrm{erg} \mathrm{cm}^{-2} \mathrm{~s}^{-1}$, which forces it to steepen dramatically. The pressure and the total energy in the chromosphere and the transition region are enhanced by a maximum factor of about 8 to 10 over their initial values. The maximum chromospheric density enhancement is by a factor of 6 . The downward propagating compression wave associated with the conduction front is eventually damped due to the increasing density as a function of depth in the chromosphere, which acts to decelerate the condensation.

Figure 3 shows the change in the apex temperature and density as a function of time for simulations $\mathrm{A}$ and $\mathrm{B}$. The ablated
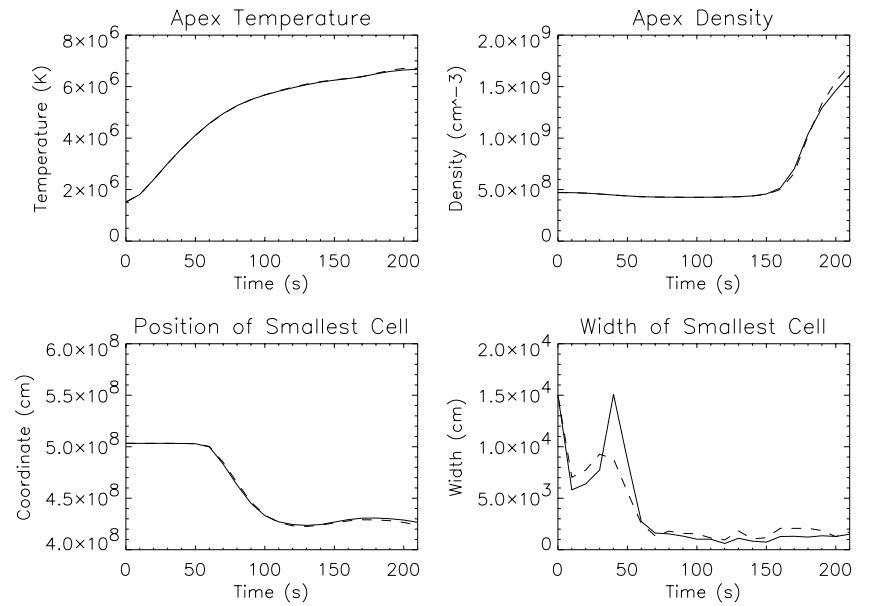

Fig. 3. Plots of apex temperature, apex density, the position of the smallest grid cell and the width of the smallest grid cell as a function of time. The results from simulation $\mathrm{A}$ are plotted as a solid line and those from simulation B are plotted as a dashed line.

material does not reach the apex of the loop until about $170 \mathrm{~s}$, at which time the density begins to increase extremely rapidly as the material ablated from each chromosphere collides. The peak density at the apex reaches a slightly greater value in simulation B than in simulation A. The physical reason for this will be discussed later.

Figure 3 also shows the response of the adaptive regridding scheme to the flare heating by plotting the position of the smallest grid cell and its size as functions of time. The qualitative behaviour is much the same in simulations A and B. Between $0 \mathrm{~s}$ and $60 \mathrm{~s}$ the coronal conduction front propagates toward the chromosphere and the position of the smallest grid cell remains fixed at the base of the transition region. At $60 \mathrm{~s}$ the conduction front reaches the base of the transition region and forces it to propagate into the chromosphere. Between $60 \mathrm{~s}$ and $100 \mathrm{~s}$ the adaptive regridding scheme tracks the transition region as it penetrates to greater depths in the chromosphere and ensures that it is always well-resolved by placing grid cells of the required size at its base. The position of the smallest grid cell remains approximately constant between $100 \mathrm{~s}$ and $210 \mathrm{~s}$. The width of the smallest grid cell fluctuates in time between $0 \mathrm{~s}$ and $40 \mathrm{~s}$ as the equilibrium solution in the footpoint region relaxes slightly, though there are clearly no quantitative changes to the physical properties of the plasma.

The smallest cell-width in both simulations is reached at about $120 \mathrm{~s}$. In simulation A it is about $6 \times 10^{2} \mathrm{~cm}$ and in simulation B about $10^{3} \mathrm{~cm}$. According to Craig et al. (1982), a heat flux on the order of $10^{8} \mathrm{erg} \mathrm{cm}^{-2} \mathrm{~s}^{-1}$ carried by the transition region requires a resolution of less than $3 \times 10^{3} \mathrm{~cm}$. In Fig. 2 the conducted heat flux peaks at just below $10^{8} \mathrm{erg} \mathrm{cm}^{-2} \mathrm{~s}^{-1}$. Therefore, the results from HYDRAD represent a substantial improvement over earlier work since the transition region structure has been successfully resolved on the required spatialscales of just a few metres, rather than kilometres. 
Table 1. Peak population fractions and corresponding temperatures, in equilibrium, for the selected transition region ions.

\begin{tabular}{ccc}
\hline \hline Ion & $Y_{i}$ & $\log _{10} T_{\text {peak }}$ \\
\hline He I & 1.00 & 4.0 \\
He II & 0.98 & 4.7 \\
C III & 0.86 & 4.8 \\
C IV & 0.34 & 5.0 \\
\hline
\end{tabular}

\subsection{Transition region ion populations and radiative emission}

The behaviour of the most strongly emitting ions can be investigated with HYDRAD and the consequences of nonequilibrium effects for the observable line intensities and the overall radiative emission can be assessed. The transition region ions selected for the current study, the peak population fractions and corresponding temperatures, in equilibrium, are given in Table 1.

\section{(a) Helium}

In equilibrium, the population fraction of He I decreases from 1 to become almost negligible over a temperature interval of $10^{4.3} \leq T \leq 10^{4.7} \mathrm{~K}$ and He II mostly exists over a temperature interval of $10^{4.4} \leq T \leq 10^{5.1} \mathrm{~K}$.

Figure 4 shows that at $60 \mathrm{~s}$ the conduction front reaches the chromosphere and the emissivities of He I and He II in simulation $\mathrm{B}$ are increased by an order of magnitude in comparison with simulation A. This is extremely significant as there are well-known intensity enhancements of helium emission lines in the solar atmosphere (Jordan 1975) that have been difficult to account for. Smith \& Jordan (2002) found that when non-thermal motions in the transition region carry helium ions across the strong temperature gradient and expose them to collisional excitation by high temperature electrons, the observed intensities of the resonance lines of He I and He II can be explained. A similar effect is operating in simulation B, though in this case the ions are stationary and the conduction front is propagating through the plasma, exposing the slowly ionising He I and He II to high temperatures, and thus enhancing the intensities of the resonance lines.

The decrease in the emissivities of $\mathrm{He} \mathrm{I}$ and $\mathrm{He}$ II after $90 \mathrm{~s}$ is due to the downflows that transport He III from higher temperature regions, while carrying $\mathrm{He}$ I and He II into the dense chromosphere where He II quickly recombines and where He I emits only weakly. By 120 s the He I and He II ionisation fronts have actually propagated farthest in simulation $\mathrm{B}$, which demonstrates the potential importance of accounting for convective transport when calculating ion populations.

$\mathrm{He}$ II is one of the strongest emitters at $10^{5} \mathrm{~K}$ (the peak of the optically-thin radiative loss function) and this depletion in its population results in a significant decrease in the overall helium emission.

\section{(b) Carbon}

Figure 5 shows the response of the C III and C IV populations and their associated emissivities as the conduction front reaches and heats the chromosphere. The emission from C III $\left(10^{4.3} \leq T \leq 10^{5.2} \mathrm{~K}\right)$ peaks at about $10^{4.9} \mathrm{~K}$ and the emission from C IV $\left(10^{4.7} \leq T \leq 10^{5.5} \mathrm{~K}\right)$ peaks at about $10^{5.1} \mathrm{~K}$ in equilibrium.

At 60 s C III exhibits qualitatively the same behaviour as He II. There is close agreement between simulations A and B in terms of the peak population fraction and the same broadening of the population distribution toward higher temperatures, as a result of the non-zero ionisation time-scale, is evident. The agreement also remains close at $80 \mathrm{~s}$, though the C III ionisation front clearly trails the conduction front in simulation $\mathrm{B}$.

By $90 \mathrm{~s}$ there is a substantial decrease in the population of C III in simulation B and by $120 \mathrm{~s}$ the ionisation front has again propagated to a greater depth than in simulation $\mathrm{A}$. The $\mathrm{C}$ III emissivity profiles follow the behaviour of the ion populations at the corresponding times and the total C III emissivity is reduced by a factor of 4 at $120 \mathrm{~s}$ in simulation B compared with its value in simulation $\mathrm{A}$.

The behaviour of C IV is rather different. At $60 \mathrm{~s}$ the population of C IV is greater by a factor of 2 in simulation $B$ than in simulation $\mathrm{A}$, with a corresponding increase in its emissivity. A factor of 2 enhancement of the population of C IV, under similar circumstances, was also found by Sarro et al. (1999). At later times the population of C IV dramatically decreases in simulation $\mathrm{B}$, becoming a factor of 3 less than in simulation $\mathrm{A}$ between $90 \mathrm{~s}$ and $120 \mathrm{~s}$. As with He II the depletion of C III and C IV is due to the downflows that transport higher ionisation stages from further up the loop, while carrying lower ionisation stages into the chromosphere where they quickly recombine. Again, this depletion in the populations of such strongly emitting ions results in a significant decrease in the overall carbon emission.

One question naturally arises: why is there such a large enhancement in the population of C IV at $60 \mathrm{~s}$ ? The answer lies in the shape of the equilibrium population distribution. The C IV population distribution is asymmetric, with a high temperature wing that extends significantly further up the loop than that of C III. In fact, asymmetric distributions, with a high temperature wing, are a common feature of Li-like ion populations. For example, the population distributions of O VI and Si XII also possess such a distribution. In simulation B ions are transported from the high temperature wing of the distribution, by the downflows, and are added to the peak population. Thus, a large enhancement of the ion population ensues, with a corresponding increase in the emissivity.

These results provide further evidence that a substantial increase or spike in the intensity of C IV (or indeed any Li-like ion) emission, over and above that which one might expect from density effects alone, could act as a signature for the presence of non-equilibrium ion populations.

\section{(c) Peak emissivities as a function of time}

Figure 6 shows the peak emissivities of carbon and helium, together with oxygen and magnesium for the purpose of comparison. An initial increase in the peak emissivity can be expected from the transition region ions as the plasma is heated by the downward propagating conduction fronts. However, the 
Table 2. Peak population fractions and corresponding temperatures, in equilibrium, for the selected coronal ions.

\begin{tabular}{ccc}
\hline \hline Ion & $Y_{i}$ & $\log _{10} T_{\text {peak }}$ \\
\hline Fe VIII & 0.43 & 5.6 \\
Fe IX & 0.71 & 5.8 \\
Fe X & 0.32 & 6.0 \\
Fe XII & 0.29 & 6.1 \\
Fe XIII & 0.35 & 6.2 \\
Fe XIV & 0.22 & 6.3 \\
Fe XV & 0.24 & 6.3 \\
Fe XVI & 0.28 & 6.4 \\
Fe XVII & 0.69 & 6.6 \\
Fe XVIII & 0.28 & 6.8 \\
Fe XIX & 0.22 & 6.9 \\
\hline
\end{tabular}

overall tendency for the transition region ions that emit close to the peak of the optically-thin radiative loss curve to become depleted at later times, when non-equilibrium effects are taken into account, acts to reduce the peak emissivity.

Recall that in Fig. 3 the peak density at the apex reaches a slightly greater value in simulation B than in simulation A. The physical reason for this is due to the supressed radiative emission in simulation B. The additional energy provided by the conducted heat flux cannot be radiated as efficiently, consequently convection is enhanced and the upflows reach higher velocities. Thus, more material is ablated into the corona.

\subsection{Coronal ion populations and radiative emission}

The coronal ions selected for the current study are all different charge states of iron since it is by far the strongest emitter at coronal temperatures. The particular ions, peak population fractions and corresponding temperatures are listed in Table 2. The choice of ions provides comprehensive temperature coverage between $10^{5.8}$ and $10^{6.9} \mathrm{~K}$. The ability to follow successive ionisation stages of elements as the plasma evolves provides a powerful tool for spectroscopic diagnostics, and relating the ionisation history of the plasma to its current ionisation state provides useful clues to understanding its evolution and properties. The usefulness of this approach will become particularly apparent when the non-equilibrium behaviour of Fe XIV to Fe XIX is investigated.

\section{(a) Fe IX to Fe XIII}

Figure 7 shows enhanced peak population fractions at $60 \mathrm{~s}$ in simulation B for all of these ions, except Fe IX, and a broadening of the population distributions toward higher temperatures, which increases with the ionisation stage. By $90 \mathrm{~s}$ the upper coronal region is entirely depleted of Fe IX to Fe XIII in both simulations. At $210 \mathrm{~s}$ in the cooler and denser regions of the loop, the population distributions of Fe IX and Fe X are in fairly close agreement between simulations A and B. However, the peak population fractions of Fe XII and Fe XIII in simulation $\mathrm{B}$ are lower than their equilibrium values.
Figure 9 shows the total Fe IX to Fe XIII (excluding Fe XI) emissivity profile for simulations A and B. At 60 s the emissivity is clearly increased in simulation B and exhibits a fairly broad plateau at around $s=10^{9} \mathrm{~cm}$, compared with its more sharply peaked profile in simulation A. However, as expected from the behaviour of the ion populations, the emission from the cooler and denser regions of the loop remains in equilibrium. By $210 \mathrm{~s}$ the entire emissivity profile in simulation B is close to equilibrium.

\section{(b) Fe XIV to Fe XVII}

Figure 8 follows the ionisation of successive stages of iron from Fe XIV to Fe XVII, between $0 \mathrm{~s}$ and $210 \mathrm{~s}$. The ionisation fronts of the higher stages follow the lower stages as they propagate down the loop. At $60 \mathrm{~s}$, in simulation $\mathrm{B}$, the populations of both Fe XIV and Fe XV are substantially enhanced over their equilibrium distributions in the upper corona. The difference between the Fe XIV populations at $s=2 \times 10^{9} \mathrm{~cm}$ $\left(T \approx 3.5 \times 10^{6} \mathrm{~K}\right)$ is a factor of 28 . The difference between the Fe XV populations at the same location is a factor of 6 . The Fe XIV and Fe XV populations are considerably depleted below $s=10^{9} \mathrm{~cm}$, compared with their equilibrium distributions. The population of Fe XVI at $60 \mathrm{~s}$ in simulation B is also enhanced toward higher temperatures and depleted toward lower temperatures compared with its equilibrium distribution.

The behaviour of Fe XVII in simulation B is markedly different, as its population at $60 \mathrm{~s}$ is significantly depleted everywhere along the loop in comparison with its equilibrium distribution. The slight bump in the Fe XVII population at $s=2 \times 10^{9} \mathrm{~cm}$ in simulation B is coincident with the peak downflow velocity and could therefore be due to the transport of Fe XVII from further up the loop. The reason for the substantial departure of Fe XVII from its equilibrium distribution can be easily understood in terms of the behaviour of the lower ionisation stages, which, as mentioned above, demonstrates the usefulness of the capability to follow the ionisation history of the plasma. The enhanced populations of Fe XIV, Fe XV and Fe XVI at $60 \mathrm{~s}$ in simulation B have consequences for the populations of the higher ionisation stages. Clearly, they can't all be enhanced because of the requirement that the total number of ions belonging to a particular element must be conserved. Therefore, an enhancement of the lower ionisation stages must result in a depletion of the higher ionisation stages at the same location.

At later times the populations of Fe XIV to Fe XVII exhibit significant departures from equilibrium, though as the coronal density increases, due to the ablation of chromospheric material, the population distributions in simulations A and B begin to agree more closely. At $210 \mathrm{~s}$, when the coronal density has increased by a factor of 3 , the population distributions of Fe XIV, Fe XV and Fe XVI are in good agreement between simulations $\mathrm{A}$ and $\mathrm{B}$. The population distribution of Fe XVII in simulation $\mathrm{B}$ is near equilibrium in the cooler and denser regions of the loop, below $s=10^{9} \mathrm{~cm}$, and it is enhanced in the upper coronal region.

Figure 9 also shows the total emissivity profiles for Fe XIV to Fe XVII. At $60 \mathrm{~s}$ the emissivity is considerably greater 
toward higher temperatures in simulation B than in simulation $\mathrm{A}$, and significantly reduced toward lower temperatures. At later times in simulation B the emissivity profile approaches equilibrium toward lower temperatures, as the density increases, though remains slightly increased toward higher temperatures. This is a result of the greater proportion of more strongly emitting lower ionisation stages in simulation B.

\section{(c) Fe XVIII to Fe XIX}

The top four panels of Fig. 10 show the population distributions of Fe XVIII and Fe XIX from $160 \mathrm{~s}$ to $210 \mathrm{~s}$ for simulations A and B. In simulation A the population of Fe XVIII is significant in the upper coronal region of the loop and does not change appreciably between $160 \mathrm{~s}$ and $210 \mathrm{~s}$. In contrast, the population of Fe XVIII in simulation B remains negligible until $190 \mathrm{~s}$, at which time the ionisation rate increases sufficiently, due to the ablated chromospheric material filling the corona, that the population of Fe XVIII begins to increase. The differences in the Fe XIX population profiles between simulations A and B are especially interesting because Fe XIX makes no appearance at all in simulation B.

This is of particular relevance because Fe XIX contains an important flare line (592 $⿱$ ) and were it not to be observed during a flare, then, under the assumption that the ion populations remain in equilibrium, one might conclude that much lower temperatures than those characteristic of Fe XIX emission were reached. In reality, however, simulation B has demonstrated that extremely high temperatures could be accompanied by emission characterised by relatively low temperature lines. Furthermore, if emission from Fe XIX is observed, then, based upon the assumption of an equilibrium ionisation balance, one might also conclude that the plasma temperature is much cooler than in reality. This is because significantly higher temperatures need to be reached in simulation B before the population of Fe XIX becomes significant.

The behaviour of successive ionisation stages in simulation $\mathrm{B}$ also indicates that the departures from equilibrium become larger with increasing ionisation stage, so these considerations may be even more important for understanding the flare lines emitted by Fe XXIV, for example. The reason for this is that line intensities generally tend to increase with increasing temperature and, since the time-scales for excitation and decay are shorter than for ionisation and recombination, the intensities of lines emitted by Fe XXIV could be underestimated, particularly if the peak non-equilibrium ion population is greater than the peak equilibrium ion population. Furthermore, for the same reason, the intensity ratio of two lines emitted by an ion of high charge state would yield plasma temperatures much greater than the equilibrium formation temperature of the emitting ion.

\section{(d) Total iron emissivity}

The bottom two panels of Fig. 10 show the total iron emissivity profile for simulations $\mathrm{A}$ and $\mathrm{B}$. The greater proportion of lower ionisation stages to higher stages in the coronal region that arises in simulation B acts to substantially enhance the emissivity over that in simulation A for the first 90 s. After $90 \mathrm{~s}$, as the density begins to increase toward lower coronal temperatures due to the chromospheric evaporation, the emissivity profiles in simulation B tend toward equilibrium and by $210 \mathrm{~s}$ they match almost exactly with simulation A.

However, at later times and higher coronal temperatures the density does not increase as rapidly because the ablated material takes longer to reach the upper regions of the loop. At $210 \mathrm{~s}$, in simulation $\mathrm{B}$, the emissivity at the apex remains slightly enhanced over its equilibrium value, despite the significant local density increase as the material ablated from each chromosphere collides.

\section{The gradual phase}

\subsection{The dynamical evolution of the plasma}

The gradual phase of the flare, for the purposes of the current study, is defined as the period of evolution after the peak temperature has been reached (Cargill et al. 1995). In Sect. 3 this was seen to be $210 \mathrm{~s}$ after the impulsive heating mechanism was switched on. Figures 11 to 13 show the evolution of the flare plasma in terms of its temperature, velocity and density, between $300 \mathrm{~s}$ and $8000 \mathrm{~s}$ (5000 s for simulation B due to time constraints), as it cools from its peak temperature of $7 \times 10^{6} \mathrm{~K}$ and gradually returns to hydrostatic equilibrium.

Between $300 \mathrm{~s}$ and $800 \mathrm{~s}$ the coronal region of the loop cools to approximately its initial temperature of $1.5 \times 10^{6} \mathrm{~K}$ at the apex. The rate of cooling is greater in simulation A than in simulation B because the non-equilibrium processes act to suppress the radiative losses from the transition region.

At $300 \mathrm{~s}$ the upflows remain substantial and reach almost $10^{7} \mathrm{~cm} \mathrm{~s}^{-1}$. The maximum velocity in simulation $\mathrm{B}$ is greater than in simulation $\mathrm{A}$ as a consequence of the suppressed radiative emission. The work of Antiochos \& Krall (1979), Doschek et al. (1982) and Gan \& Fang (1990), discussed in Sect. 1, also found that the ablation continued well into the gradual phase and significantly increased the coronal density. The results from HYDRAD are in good agreement with this previous work and are consistent with observations, which show the peak in the emission measure from a flaring loop is reached after the peak in temperature.

By $500 \mathrm{~s}$ material has begun to drain out of the upper coronal region, reaching peak downflow velocities of almost $4 \times 10^{6} \mathrm{~cm} \mathrm{~s}^{-1}$ at $700 \mathrm{~s}$ in simulation $\mathrm{A}$ and just under $3 \times 10^{6} \mathrm{~cm} \mathrm{~s}^{-1}$ in simulation B. At $800 \mathrm{~s}$ the loop reaches its initial apex temperature, though the coronal density is far greater than at $t=0 \mathrm{~s}$. It was also shown in BM1 that loops tend to cool more quickly than they drain. The over-density of loops observed by TRACE at temperatures around $10^{6} \mathrm{~K}$, compared with hydrostatic equilibrium models, could thus be explained by cooling from much greater temperatures after a period of chromospheric evaporation, in agreement with the findings of Warren et al. (2002) and Spadaro et al. (2003).

One problem remains: the persistence of these loops for periods longer than the characteristic cooling time (Lenz et al. 1999 ) is difficult to explain satisfactorily, though it seems likely that it could be accounted for by a more slowly decaying rate of heating. However, theoretically predicted cooling times of 
compact flares are in reasonably good agreement with observationally measured values and so it is unclear whether additional heat input during the gradual phase is necessary (Wu et al. 1986).

Beyond $800 \mathrm{~s}$ the catastrophic cooling of coronal material to transition region temperatures takes place. Catastrophic cooling after a strong heating event was also shown by Spadaro et al. (2003). In simulation A the total cooling time from the peak temperature at $210 \mathrm{~s}$ to the minimum temperature reached at $1400 \mathrm{~s}$ is $1190 \mathrm{~s}$, or $19.8 \mathrm{~min}$, which is in excellent agreement with the cooling time of 20 min found by Gan \& Fang (1990).

By $1400 \mathrm{~s}$ the downflows have drained sufficient material from the coronal region that it is close to its initial density and so the constant background heating begins to recover the initial hydrostatic equilibrium. Between $1600 \mathrm{~s}$ and $8000 \mathrm{~s}$ the temperature and the density return to their initial values everywhere along the loop. The plasma flows gradually decay away, eventually becoming negligible in comparison with the sound speed and have no further influence upon the evolution of the loop.

\subsection{Transition region ion populations and radiative emission}

\section{(a) Helium}

Figures 14 and 15 show the population distributions and the total emissivity of helium between $300 \mathrm{~s}$ and $1400 \mathrm{~s}$, as the loop cools from its peak apex temperature of $7 \times 10^{6} \mathrm{~K}$ to temperatures below $5 \times 10^{5} \mathrm{~K}$.

The population fractions of He I and He II are everywhere reduced in simulation $\mathrm{B}$ when compared with simulation A. The population fraction of He I is effectively 1.0 in the low temperature, high density chromosphere in both simulations, though the gradient across the ionisation front is much less severe at later times in simulation $\mathrm{B}$. The erosion of the strong gradients and the broadening of the population distributions is a consequence of the transportation of ions by the bulk flows. Convection generally acts to smooth out gradients and discontinuities.

The total emissivity profile of helium tells a similar story to the ion population profiles. The peak emissivity is considerably lower at all times in simulation B and extends to greater heights. This reduced emissivity is one of the main contributors to the differences in the radiative cooling time-scales and the bulk flow velocities that arise between the simulations.

\section{(b) Carbon}

Figures 16 and 17 show the population distributions and the total emissivity of C III and C IV during the gradual phase of the flare. As for helium, the population fractions remain strongly reduced in simulation $\mathrm{B}$. In fact, the magnitude of the reduction is over a factor of 4 at most times for both C III and C IV. This has clear implications for the theoretical calculation of spectral line intensities.
The total C III and C IV emissivity profiles demonstrate the effect of the severely depleted ion populations. They are reduced by almost an order of magnitude in simulation B, compared with the corresponding emissivities in simulation A. As for helium, the decreased emissivity has extremely important consequences for the radiative cooling time-scale and the bulk flow velocity of the plasma. The C III and C IV emissivity profiles, in common with He I and He II, are also broadened when the lowest temperatures are reached, though the spatial extent of the broadening is considerably greater in simulation A.

It is interesting to note that while the peak emissivity of C III and C IV is larger than that of He I and He II, the spatial extent of the latter is greater at all times in both simulations. Between $300 \mathrm{~s}$ and $800 \mathrm{~s}$ the emissivity of the carbon ions is strongly peaked, whereas the emissivity of the helium ions is relatively broad. Between $900 \mathrm{~s}$ and $1400 \mathrm{~s}$ the emissivity profile of the carbon ions broadens slightly, but remains considerably narrower than that of the helium ions. The somewhat surprising consequence of this is that He I and He II emit more strongly further up the loop, at higher temperatures, than C III and C IV. For example, in simulation B at $1400 \mathrm{~s}$ and $s=4.5 \times 10^{8} \mathrm{~cm}$, the total helium emissivity is around $10^{-23.4} \mathrm{erg} \mathrm{cm}^{3} \mathrm{~s}^{-1}$, whereas the total C III and C IV emissivity has fallen far below this amount. The same effect is present in simulation A, though to a far lesser extent. Therefore, given sufficient spatial resolution, it is possible that one may observe significant emission from helium overlying emission from carbon, from the same magnetic structure in the presence of a nonequilibrium ionisation balance.

\section{(c) Total emissivity}

Figure 18 shows the total emissivity profiles in the left-hand footpoint region of the loop between $300 \mathrm{~s}$ and $1400 \mathrm{~s}$ for simulations $\mathrm{A}$ and $\mathrm{B}$. The differences in the emissivity profiles are exactly as expected from the discussion above. The peak radiative emission at lower transition region temperatures is strongly reduced at all times in simulation B compared with simulation A. At $300 \mathrm{~s}$ the total emissivity at upper transition region temperatures is slightly enhanced in simulation B because of the greater abundance of more strongly emitting lower ionisation stages, though by $800 \mathrm{~s}$ the total emissivity curves agree more closely in this region.

Between $900 \mathrm{~s}$ and $1400 \mathrm{~s}$ the peak of the total emissivity curve in simulation A broadens considerably due to the behaviour of ions such as C III and C IV, discussed above. However, the total emissivity profiles in the upper region of the footpoint remain in fairly good agreement. A significant contribution to the total emissivity in the upper footpoint region is made by the higher ionisation stages such as $\mathrm{C} \mathrm{V}$, which have fewer stages to recombine through as the plasma cools. It was shown in BM1 that the very highest ionisation stages persist for the longest periods of time as the plasma cools, which results in the depletion of the lower ionisation stages and consequently reduces the overall radiative emission. However, the coronal densities in the post-flare plasma are greater and so the populations of these ions are able to remain closer to equilibrium. 


\subsection{Coronal ion populations and radiative emission}

\section{(a) Fe VIII to Fe XII}

Figures 19 and 20 show the population distributions of the upper transition region and coronal ions Fe VIII, Fe IX, Fe X and Fe XII between $300 \mathrm{~s}$ and $1400 \mathrm{~s}$ for simulations A and B. As the plasma cools between $300 \mathrm{~s}$ and $800 \mathrm{~s}$ the population distributions of Fe VIII and Fe IX broaden. The peak population of Fe VIII descends the loop, whereas the peak population of Fe IX ascends the loop. The differences between simulations A and $\mathrm{B}$ can be reasonably pronounced, with the population fraction of Fe IX at $800 \mathrm{~s}$ and $s=2 \times 10^{9} \mathrm{~cm}$ a factor of 3 greater in simulation A than in simulation B. However, as the density decreases further down the loop the differences between simulations $\mathrm{A}$ and $\mathrm{B}$ in the vicinity of the peak populations become relatively small.

The Fe X and Fe XII population distributions also broaden between $300 \mathrm{~s}$ and $800 \mathrm{~s}$, although toward higher temperatures only and the peak population fractions ascend the loop. At $800 \mathrm{~s}$ the apex population of $\mathrm{Fe} \mathrm{X}$ is a factor of 4 greater in simulation $\mathrm{A}$ than in simulation $\mathrm{B}$. The peak population of Fe XII increases slightly with time in simulation B because the downflows are sufficiently strong to allow convection to make a contribution.

Between $900 \mathrm{~s}$ and $1400 \mathrm{~s}$ the population of Fe VIII increases and the peak population ascends the loop slightly as lower ionisation stages become more abundant further down. There is a significant amount of Fe IX present in both simulations where the temperatures have fallen to values around $5 \times 10^{5} \mathrm{~K}$. The population of Fe IX is greater in simulation B and a small amount of $\mathrm{Fe} X$ persists.

The enhanced presence in relatively cool plasma of ions that are commonly assumed to be most abundant at coronal temperatures may be sufficient to increase the intensity of their emission above equilibrium values and, furthermore, may lead one to overestimate the temperature of the plasma when an equilibrium ionisation balance is assumed.

Figure 21 shows the total Fe VIII, Fe IX, Fe $X$ and Fe XII emissivity profiles between $300 \mathrm{~s}$ and $1400 \mathrm{~s}$ for simulations A and B. As the loop begins to cool, the higher ionisation stages (such as Fe XIV to Fe XVIII) dominate the contribution to the emissivity. The lower ionisation stages (such as Fe VIII to Fe XII) gradually become more important as lower temperatures are reached and dominate by $800 \mathrm{~s}$.

\section{(b) Total emissivity}

Figure 22 shows the total emissivity in the coronal region of the loop between $300 \mathrm{~s}$ and $1400 \mathrm{~s}$. The total emissivity qualitatively follows the behaviour of the selection of ions already discussed and can be understood in terms of these. At $300 \mathrm{~s}$ the total emissivity is greater in simulation B because of the enhancement in the population of lower ionisation stages, compared with simulation A, which emit more strongly.

At $800 \mathrm{~s}$ there is an enhanced population of more weakly emitting higher ionisation stages in simulation $\mathrm{B}$, due to the finite recombination time-scale, and so the total emissivity is greater in simulation $\mathrm{A}$. The total emissivity remains greater in simulation A after $800 \mathrm{~s}$, as can be seen in the bottom two panels of Fig. 22. As time progresses and the loop relaxes back toward its initial hydrostatic equilibrium configuration, the differences between simulations A and B become negligible.

\section{Observational consequences for TRACE}

Figure 23 shows profiles of the synthesised counts in the $171 \AA$ and $195 \AA$ wavelength bands of TRACE at key stages during the impulsive and the gradual phases of the compact flare. The counts in the $171 \AA$ range are mostly due to emission from Fe IX and Fe X, though we also include lines from O IV, O V and O VI. The 195 A range is dominated by Fe VIII and Fe XII, with contributions from $\mathrm{O} I V$ and $\mathrm{O} V$. In order to sythesise the counts an estimate of the line-of-sight depth of the emitting plasma is required. We adopt a value of $5 \times 10^{8} \mathrm{~cm}$, which is similar to the observationally measured value of $5.8 \times 10^{8} \mathrm{~cm}$ given by Del Zanna \& Mason (2003).

The temperatures attained along most of the coronal region of the loop during the impulsive phase exceed the upper limit to the range of sensitivity of both filters. However, the transition region and lower coronal region of the loop remain brightly visible, so the loop would perhaps be observed as a vertical structure with no apparent overlying emission if viewed from the side, or as a patch of emission on the order of $10^{6} \mathrm{~K}$ if viewed from above, especially if there were several footpoints of similarly flaring loops embedded within the same region of strong magnetic field. The nature of the so-called active region moss observed by TRACE (Berger et al. 1999; Fletcher \& de Pontieu 1999) is akin to the description of the latter.

At $90 \mathrm{~s}$ the predicted peak intensities of emission detected by both filters increase by over an order of magnitude compared with their values at $t=0 \mathrm{~s}$ in simulations $\mathrm{A}$ and $\mathrm{B}$. The maximum and minimum in emission detected by the $195 \AA$ filter are predicted by simulation $B$ to be closer in value. The deeper minimum in simulation $\mathrm{A}$ is due to the instantaneous ionisation of Fe VIII, whereas in simulation B the ionisation time-scale is non-zero. The enhanced maximum in simulation $\mathrm{A}$ is due to the instantaneous ionisation of lower stages to Fe XII. The predicted emission detectable by both filters also extends further along the loop in simulation $\mathrm{B}$ due to the non-zero ionisation time-scales of Fe IX, Fe X and Fe XII. At $210 \mathrm{~s}$ and $400 \mathrm{~s}$ the maxima and minima in both filters are in very close agreement between simulations $\mathrm{A}$ and $\mathrm{B}$, which is due to the enhanced densities resulting from the chromospheric evaporation. The detectable emission in both filters extends further along the loop at $210 \mathrm{~s}$ in simulation B, though at $400 \mathrm{~s}$ the location of the cut-off is in almost exact agreement between simulations $\mathrm{A}$ and $\mathrm{B}$.

At $800 \mathrm{~s}$ the predicted emission detected by the $171 \AA$ filter is reduced in simulation B along most of the coronal region of the loop due to the delay in recombination to Fe IX and Fe X. The predicted emission detected by the $195 \AA$ filter is in relatively close agreement between simulations A and B because the delay in recombination to Fe XII is somewhat less. At $1400 \mathrm{~s}$ the predicted emission in the $171 \AA$ filter is greater in simulation B due to the delay in the recombination of Fe IX and $\mathrm{Fe} \mathrm{X}$ to lower ionisation stages. The predicted emission in 
the $195 \AA$ filter is also greater and extends further along the loop in simulation $\mathrm{B}$ due to the delay in the recombination of Fe VIII. The results of simulation B at $1400 \mathrm{~s}$ and $T \approx 5 \times 10^{5} \mathrm{~K}$ (along most of the coronal region of the loop), show that more intense emission from ions such as Fe IX and Fe X than would be predicted by a conventional equilibrium treatment, can be observed from plasma at temperatures well below their formation temperatures. In fact, the intensity of emission in the $171 \AA$ filter at $1400 \mathrm{~s}$ is actually greater than at $t=0 \mathrm{~s}$ along the upper part of the loop. The persistence of the loop in the TRACE filters at these relatively low temperatures might go some way toward explaining the longevity of cooling loops observed by TRACE. If they remain visible at such low temperatures and subsequently during the recovery of their initial equilibrium after a thermal perturbation, then the longevity of the observed loops can be reconciled with the time-scales involved in this process. The loops themselves may only disappear from view when some other process, perhaps related to the magnetic field, acts to destabilise them.

Forward modelling the detectable emission in the TRACE wavelength bands in the manner carried out here provides a very powerful tool. The consequences of assuming an equilibrium ionisation balance when deriving temperatures using the filter ratio technique can be investigated and the potential for misinterpreting observational data assessed.

Figure 24 shows profiles of the predicted filter ratios for simulations $\mathrm{A}$ and $\mathrm{B}$, which exhibit clear differences and will lead to discrepancies between the actual and derived plasma temperatures. At $90 \mathrm{~s}$ the temperature at $s=8 \times 10^{8} \mathrm{~cm}$ is about $2.1 \times 10^{6} \mathrm{~K}$ (Fig. 2), however, assuming that simulation B describes the properties of the observed loop the filter ratio technique yields a value of $1.7 \times 10^{6} \mathrm{~K}$. The ratio, as a function of temperature, of the response functions of the $171 \AA$ and $195 \AA$ filters was taken from Del Zanna \& Mason (2003), who use atomic data from version 4.01 of the CHIANTI database (Young et al. 2003; Dere et al. 1997), the ionisation balance of Mazzotta et al. (1998), coronal element abundances and the TRACE ground calibration.

At $210 \mathrm{~s}$ the temperature at $6 \times 10^{8} \mathrm{~cm}$ is about $1.8 \times 10^{6} \mathrm{~K}$, whereas the filter ratio technique yields two possible temperatures of $1.58 \times 10^{6} \mathrm{~K}$ and $2.24 \times 10^{6} \mathrm{~K}$. This is because the filter ratio is close to a local maximum in the curve of the ratio of the filter response functions versus temperature. Differences between the filter ratios at $90 \mathrm{~s}$ and $210 \mathrm{~s}$ in simulations A and B also exist further down the loop.

At $400 \mathrm{~s}$ the filter ratio curves in Fig. 24 actually exhibit relatively good agreement when one considers that the transition region propagates further into the chromosphere in simulation $\mathrm{B}$, which naturally results in a displacement of the filter ratio curve relative to the curve given by simulation $\mathrm{A}$.

At $800 \mathrm{~s}$ the differences in the filter ratio curves between simulations $\mathrm{A}$ and $\mathrm{B}$, particularly along most of the coronal region, appear to be quite pronounced. However, the high densities present actually ensure that the filter ratio temperatures derived using these curves agree relatively well with the simulated temperatures. For example, in simulation A the temperature at $4 \times 10^{9} \mathrm{~cm}$ is about $1.4 \times 10^{6} \mathrm{~K}$ (Fig. 11) and the filter ratio temperature is $1.26 \times 10^{6} \mathrm{~K}$, although a considerably higher value of $2.57 \times 10^{6} \mathrm{~K}$ could not be discounted due to the non-uniqueness of the ratio value as a function of temperature. In simulation B the temperature at $4 \times 10^{9} \mathrm{~cm}$ is about $1.5 \times 10^{6} \mathrm{~K}$ and the filter ratio temperature is $1.45 \times 10^{6} \mathrm{~K}$, although, again, a value of $2.14 \times 10^{6} \mathrm{~K}$ could not be discounted from the observational data alone.

Clearly, though the filter ratio technique is not as accurate as spectroscopic techniques that use line ratios from the same ion for deriving the plasma temperature, it yields acceptable results for qualitative purposes when the assumption of an equilibrium ionisation balance is valid, with uncertainties in the derived temperature of around $10 \%$.

\section{Summary and conclusions}

HYDRAD has been used to simulate a compact flare of moderate energy, driven by a thermal mechanism depositing heat in the corona, with due account taken of the departure from equilibrium of the ion populations and their associated radiative emission. The evolution of the plasma in terms of its thermal properties, dynamics and ion populations has been followed through the impulsive phase, the gradual phase and the subsequent recovery of the initial hydrostatic equilibrium. Thus, a self-consistent analysis of the interdependent phases of a compact flare has been carried out. The results from HYDRAD have been shown to be consistent with the work of others in terms of the overall, qualitative behaviour of the system, although important quantitative differences have emerged as a consequence of the non-equilibrium processes that affect the ion populations and the radiative emission.

\section{(a) The impulsive phase}

During the impulsive phase, an ablation of chromospheric material is evident when the plasma is conductively heated, with upflows reaching peak velocities of $2 \times 10^{7} \mathrm{~cm} \mathrm{~s}^{-1}$. An oppositely directed compression wave is also observed to propagate toward the chromosphere, where it eventually becomes damped out in the high density plasma. The coronal density increases by a factor of about 3 at the apex when the ablated material in each half of the loop collides. A factor of 3 increase in the density will result in almost an order of magnitude increase in the intensity of emission since it is proportional to $n^{2}$. The adaptive regridding scheme is shown to successfully track and resolve the transition region as it steepens dramatically and propagates into the chromosphere. The smallest cell-width is only $6 \times 10^{2} \mathrm{~cm}$, which represents an improvement of several orders of magnitude over the resolution achieved in previous work.

In the lower transition region strong non-equilibrium enhancements are present in the ion populations when the conduction front reaches the chromosphere, particularly for the Li-like C IV. These enhancements are due to the extended high temperature wing of the population distribution and the downflows contributing to the peak population fraction via convection. The high temperature wing of the distribution remains slightly enhanced due to the non-zero ionisation time-scale. The emissivities of lower transition region ions such as He I, $\mathrm{He}$ II and C IV are increased by up to an order of magnitude as these ions find themselves emitting at considerably higher 
temperatures. Strong emission lines are always observed during flares, which may be due to both density enhancements and the influence of a non-equilibrium ion population. The evidence therefore suggests that accounting for non-equilibrium ion populations is necessary in order to make theoretically calculated line intensities more consistent with their observationally measured values. The inclusion of non-equilibrium effects in the present case certainly alters the predicted intensity of the He I, He II and C IV emission in the way necessary to bring them into closer agreement with observations (Jordan 1975; Del Zanna et al. 2002).

An over-abundance of lower ionisation stages compared with their equilibrium populations is evident in the corona as the plasma temperature increases. Therefore, the coronal emissivity is enhanced away from equilibrium because lower ionisation stages are generally stronger emitters at high temperatures than the higher ionisation stages that would exist there in equilibrium. The non-zero ionisation time-scale also means that the departures from equilibrium become increasingly important for higher ionisation stages because the delay between events accumulates. It has been seen that, for example, the population of Fe XIX is severely depleted at the maximum temperature reached by the plasma when non-equilibrium effects are accounted for. However, according to the equilibrium ionisation balance it should be relatively abundant.

This depletion of high ionisation stages has important implications for the analysis of observational data. The results from HYDRAD have shown that high temperatures can be reached without the corresponding emission one would expect to see in equilibrium, which could result in a severe underestimation of the temperature if non-equilibrium effects were omitted from the analysis. Furthermore, the results from HYDRAD have shown that significantly higher temperatures (than indicated by the assumption of equilibrium) need to be reached before the populations of the highest ionisation stages of iron are sufficient for their emission to become significant. This also means that measurements made using the ratio of two lines emitted from the same ion would yield significantly greater plasma temperatures than the equilibrium formation temperature of the emitting ion.

The ablation of material is seen to continue well into the gradual phase, thus verifying the results of Antiochos \& Krall (1979), Doschek et al. (1982) and Gan \& Fang (1990). Since the density continues to increase, the peak in the emission measure occurs after the peak in temperature is reached, which is consistent with observations.

\section{(b) The gradual phase}

During the gradual phase the populations of the strongest transition region emitters are substantially reduced from their equilibrium values, as are the lower ionisation stages of elements such as neon and magnesium, due to the non-zero recombination time-scale of the higher ionisation stages. Therefore, the radiative emission from the transition region is also reduced, which results in lower downflow velocities and a somewhat increased time-scale of cooling.
The total emissivity in the corona is higher than one would expect in equilibrium at the beginning of the gradual phase, due to the over-abundance of strongly emitting, lower ionisation stages of iron. It is reduced thereafter due to the greater abundance of weakly emitting, higher ionisation stages than one would expect in equilibrium.

When the loop cools to its temperature at $t=0 \mathrm{~s}$, the coronal density remains substantially enhanced over the density one would expect to find in hydrostatic equilibrium. Therefore, the cooling process after an initial dynamic heating event provides a likely explanation for the over-density of loops observed by TRACE at temperatures around $10^{6} \mathrm{~K}$. This is in close agreement with the findings of Warren et al. (2002) and Spadaro et al. (2003). However, the persistence of these loops over periods considerably greater than their characteristic cooling times remains unresolved. It appears that including non-equilibrium effects when calculating the radiative energy loss does not decrease the rate of cooling sufficiently to enhance the longevity of the loop, making only a few minutes difference at most to the cooling time. Plausible suggestions include a multi-thread loop model with individual filaments at different stages in their evolution. However, estimates of the loop filling factor (the fraction of the resolved volume of plasma actually emitting) on the order of 1.0 (Del Zanna \& Mason 2003) means that there could be too few unresolved filaments to make this explanation viable.

Alternatively, since the loop cools from Fe XII to Fe VIII in the TRACE filters and it has only recently been realised that Fe VIII is a strong emitter in the $195 \AA$ passband (Del Zanna 2003, private communication), Fe VIII emission could previously have been mistaken for hotter Fe XII emission. This would result in the overestimation of the cooling time since the loop would appear to be maintained at higher temperatures for longer. A further possibility is that after the initial impulse of energy the rate of heating could decay more slowly and provide sufficient energy to increase the cooling time. These lines of enquiry will be pursued in future work with HYDRAD.

During the final stages of the gradual phase the coronal plasma cools catastrophically to transition region temperatures, due to the enhanced density, before the downflows drain sufficient material away to allow the initial hydrostatic equilibrium to be recovered. This behaviour was also found by Spadaro et al. (2002) and Spadaro et al. (2003).

\section{(c) Observational consequences for TRACE}

Finally, the intensity of emission that would be detected by the TRACE $171 \AA$ and $195 \AA$ filters has been synthesised. During the impulsive phase only the low lying, relatively low temperature, regions of the loop would be visible with no discernible overlying emission, perhaps reminiscent of the active region moss observations of others. The filter ratio technique for the determination of temperatures has been shown to underestimate the temperature of the plasma in the low lying regions of the loop during the impulsive phase as a consequence of departures of the ionisation balance from equilibrium, which may have important implications for the observationally derived properties of moss-like regions. 
During the gradual phase, the enhanced densities ensure that the ionisation balance in simulation B remains fairly close to equilibrium and therefore the filter ratio technique may do a reasonably good job of estimating the plasma temperature under these circumstances only. As the ablated material drains back into the chromosphere the departures from equilibrium of the ionisation balance become more pronounced.

The synthesised emission in both filters shows that the loop should remain visible at much lower temperatures than would be indicated by a purely equilibrium treatment of the ion populations and the radiative emission. Therefore, the longevity of observed loops in these TRACE filters can potentially be reconciled with a non-equilibrium population of emitting ions. The loop is predicted to remain visible as it cools to low temperatures and as it recovers its equilibrium after the thermal perturbation. This process takes several hours, in agreement with the time-scales for which similar loops persist (Lenz et al. 1999), and eventually another process, perhaps related to the magnetic field, may act to destabilise the loop and cause it to disappear from view.

Acknowledgements. The authors acknowledge the financial support of PPARC and wish to thank the anonymous referee for their careful reading of the original manuscript, and for their suggestions for improvements.

\section{References}

Antiochos, S. K., \& Krall, K. R. 1979, ApJ, 229, 788

Aschwanden, M. J., \& Alexander, D. 2001, Sol. Phys., 204, 91

Berger, T. E., de Pontieu, B., Schrijver, C. J., \& Title, A. M. 1999, ApJ, 519, L97

Betta, R. M., Peres, G., Reale, F., \& Serio, S. 2001, A\&A, 380, 341

Bradshaw, S. J., \& Mason, H. E. 2003a, A\&A, 401, 699

Bradshaw, S. J., \& Mason, H. E. 2003b, A\&A, 407, 1127

Cargill, P. J., Mariska, J. T., \& Antiochos, S. K. 1995, ApJ, 439, 1034

Cheng, C.-C., Oran, E. S., Doschek, G. A., Boris, J. P., \& Mariska, J. T. 1983, ApJ, 265, 1090

Craig, I. J., Robb, T. D., \& Rollo, M. D. 1982, Sol. Phys., 76, 331

Del Zanna, G., \& Mason, H. E. 2003, A\&A, 406, 1089

Del Zanna, G., Landini, M., \& Mason, H. E. 2002, A\&A, 385, 968
Dere, K. P., Landi, E., Mason, H. E., Monsignori Fossi, B. C., \& Young, P. R. 1997, VizieR Online Data Catalog, 412, 50149

Doschek, G. A., Boris, J. P., Cheng, C. C., Mariska, J. T., \& Oran, E. S. 1982, ApJ, 258, 373

Fisher, G. H., Canfield, R. C., \& McClymont, A. N. 1985, ApJ, 289, 414

Fletcher, L., \& de Pontieu, B. 1999, ApJ, 520, L135

Fludra, A., Doyle, J. G., Metcalf, T., et al. 1995, A\&A, 303, 914

Gan, W. Q., \& Fang, C. 1990, ApJ, 358, 328

Jordan, C. 1975, MNRAS, 170, 429

Karpen, J. T., \& Devore, C. R. 1987, ApJ, 320, 904

Lenz, D. D., Deluca, E. E., Golub, L., Rosner, R., \& Bookbinder, J. A. 1999, ApJ, 517, L155

MacNeice, P. 1986, Sol. Phys., 103, 47

MacNeice, P., Burgess, A., McWhirter, R. W. P., \& Spicer, D. S. 1984, Sol. Phys., 90, 357

Masuda, S., Kosugi, T., Hara, H., et al. 1995, PASJ, 47, 677

Mazzotta, P., Mazzitelli, G., Colafrancesco, S., \& Vittorio, N. 1998, A\&AS, 133, 403

McClymont, A. N., \& Canfield, R. C. 1983, ApJ, 265, 483

Nagai, F. 1980, Sol. Phys., 68, 351

Nagai, F., \& Emslie, A. G. 1984, ApJ, 279, 896

Nagai, F., Wu, S. T., \& Tandberg-Hanssen, E. 1983, Sol. Phys., 84, 271

Pallavicini, R., Peres, G., Serio, S., et al. 1983, ApJ, 270, 270

Peres, G., Reale, F., Serio, S., \& Pallavicini, R. 1987, ApJ, 312, 895

Sarro, L. M., Erdélyi, R., Doyle, J. G., \& Pérez, M. E. 1999, A\&A, 351,721

Shibata, K., Masuda, S., Shimojo, M., et al. 1995, ApJ, 451, L83

Smith, G. R., \& Jordan, C. 2002, MNRAS, 337, 666

Spadaro, D., Lanza, A. F., Lanzafame, A. C., et al. 2003, ApJ, 582, 486

Spadaro, D., Lanza, A. F., Lanzafame, A. C., et al. 2002, in From Solar Min to Max: Half a Solar Cycle with SOHO, ESA SP-508, 331

Spitzer, L. 1962, Physics of Fully Ionized Gases (New York: Interscience), 2nd edition

Tsiklauri, D., Aschwanden, M. J., Nakariakov, V. M., \& Arber, T. D. 2004, A\&A, 419, 1149

Warren, H. P., Winebarger, A. R., \& Hamilton, P. S. 2002, ApJ, 579, L41

Wu, S. T., de Jager, C., Dennis, B. R., et al. 1986, in Energetic Phenomena on the Sun, ed. M. R. Kundu, \& B. E. Woodgate, NASA Conf. Publ., 2439, 5

Young, P. R., Del Zanna, G., Landi, E., et al. 2003, ApJS, 144, 135 


\section{Online Material}


S. J. Bradshaw et al.: Compact flare emission, Online Material p 2
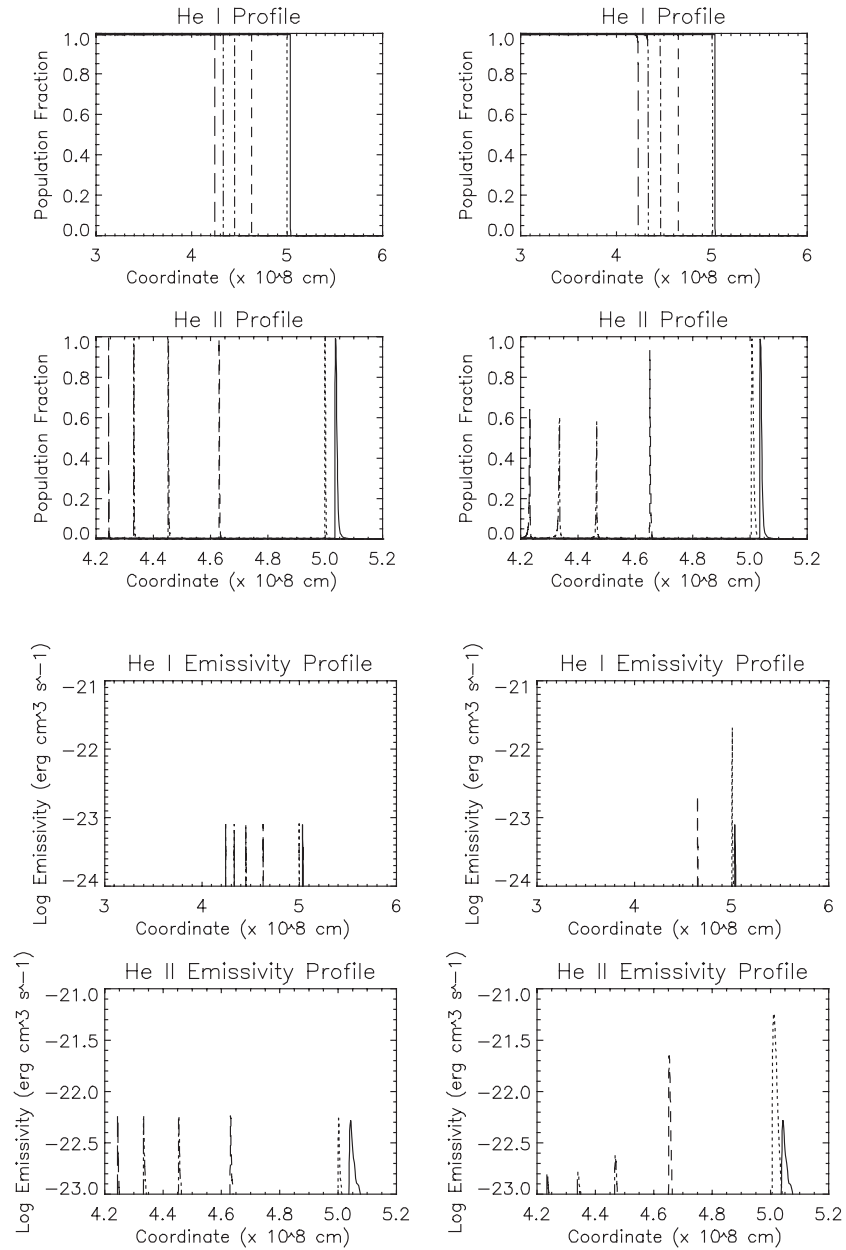

Fig. 4. Time series of the He I and He II population fractions and emissivities at: $0 \mathrm{~s}$ (solid), $60 \mathrm{~s}$ (dotted), $80 \mathrm{~s}$ (dashed), $90 \mathrm{~s}$ (dash-dot), $100 \mathrm{~s}$ (dash-dot-dot-dot) and $120 \mathrm{~s}$ (long-dashed). The left-hand panels show the results for simulation A and the right-hand panels show the results for simulation $\mathrm{B}$.
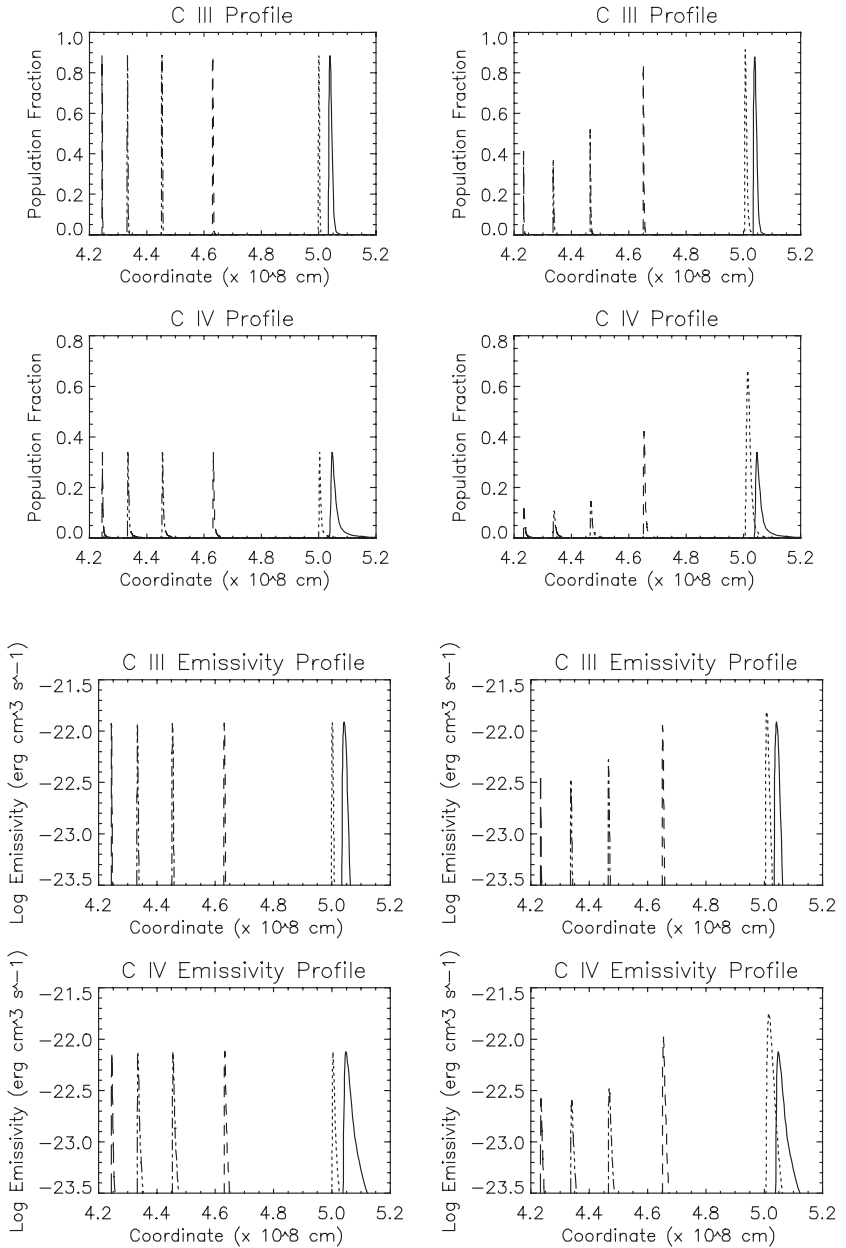

Fig. 5. Time series of the C III and C IV population fractions and emissivities at: $0 \mathrm{~s}$ (solid), $60 \mathrm{~s}$ (dotted), $80 \mathrm{~s}$ (dashed), $90 \mathrm{~s}$ (dash-dot), $100 \mathrm{~s}$ (dash-dot-dot-dot) and $120 \mathrm{~s}$ (long-dashed). The left-hand panels show the results for simulation A and the right-hand panels show the results for simulation $\mathrm{B}$.
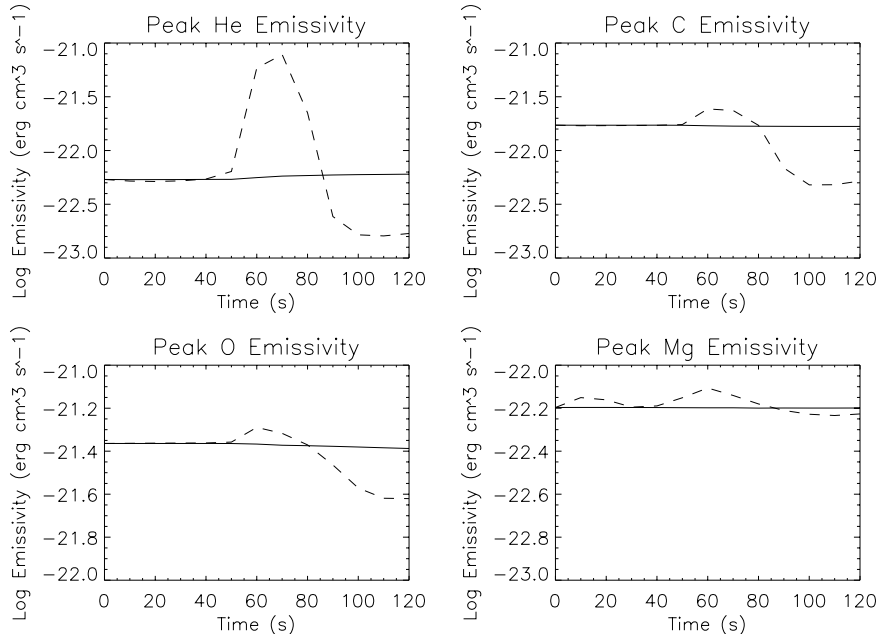

Fig. 6. Plots of the peak helium, carbon, oxygen and magnesium emissivities as a function of time. The results from simulation A are plotted as a solid line and those from simulation B are plotted as a dashed line. 
S. J. Bradshaw et al.: Compact flare emission, Online Material $p 3$
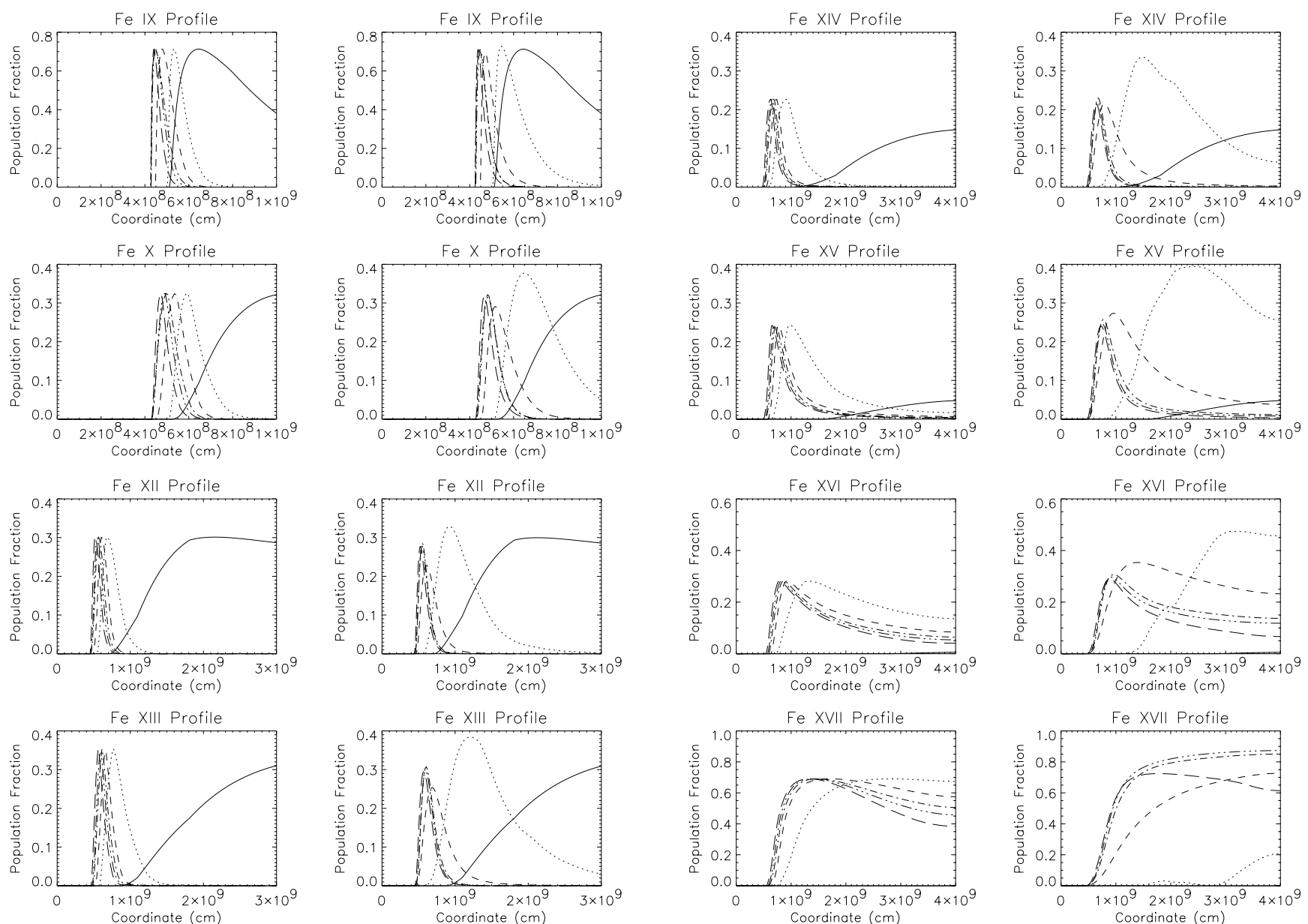

Fig. 7. Time series of the Fe IX, Fe X, Fe XII and Fe XIII population fractions at: $0 \mathrm{~s}$ (solid), $60 \mathrm{~s}$ (dotted), $90 \mathrm{~s}$ (dashed), $120 \mathrm{~s}$ (dash-dot), $150 \mathrm{~s}$ (dash-dot-dot-dot) and $210 \mathrm{~s}$ (long-dashed). The left-hand panels show the results for simulation $\mathrm{A}$ and the right-hand panels show the results for simulation $\mathrm{B}$.
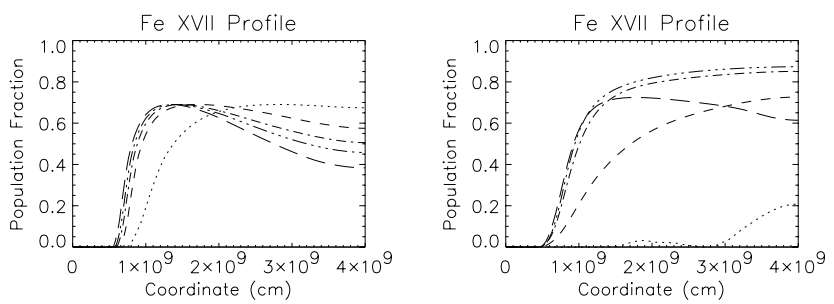

Fig. 8. Time series of the Fe XIV, Fe XV, Fe XVI and Fe XVII population fractions at: $0 \mathrm{~s}$ (solid), $60 \mathrm{~s}$ (dotted), $90 \mathrm{~s}$ (dashed), $120 \mathrm{~s}$ (dashdot), $150 \mathrm{~s}$ (dash-dot-dot-dot) and $210 \mathrm{~s}$ (long-dashed). The left-hand panels show the results for simulation $\mathrm{A}$ and the right-hand panels show the results for simulation $\mathrm{B}$.
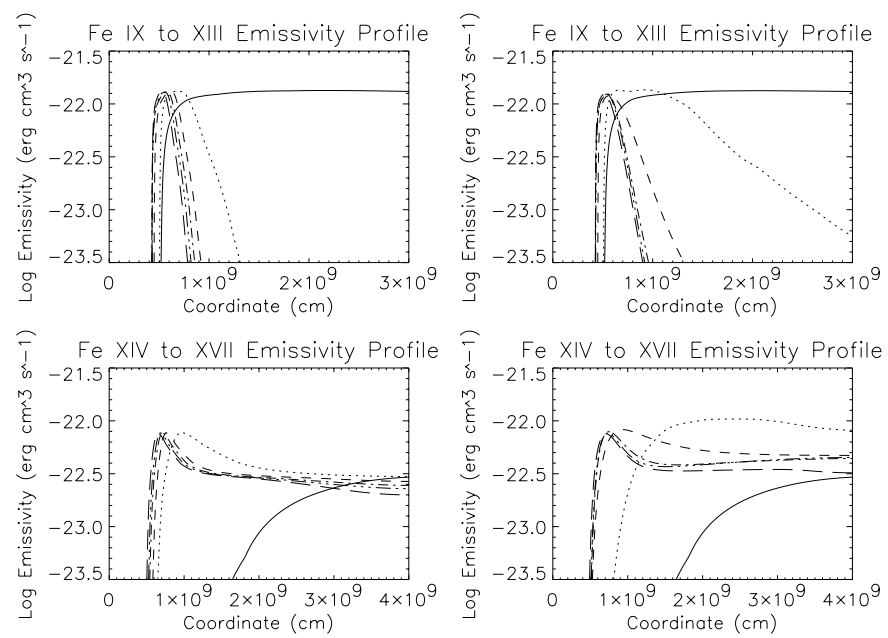

Fig. 9. Time series of the total Fe IX, Fe X, Fe XII and Fe XIII emissivity, and the total Fe XIV, Fe XV, Fe XVI and Fe XVII emissivity at: $0 \mathrm{~s}$ (solid), $60 \mathrm{~s}$ (dotted), $90 \mathrm{~s}$ (dashed), $120 \mathrm{~s}$ (dash-dot), $150 \mathrm{~s}$ (dashdot-dot-dot) and $210 \mathrm{~s}$ (long-dashed). The left-hand panels show the results for simulation $\mathrm{A}$ and the right-hand panels show the results for simulation $\mathrm{B}$. 
S. J. Bradshaw et al.: Compact flare emission, Online Material p 4
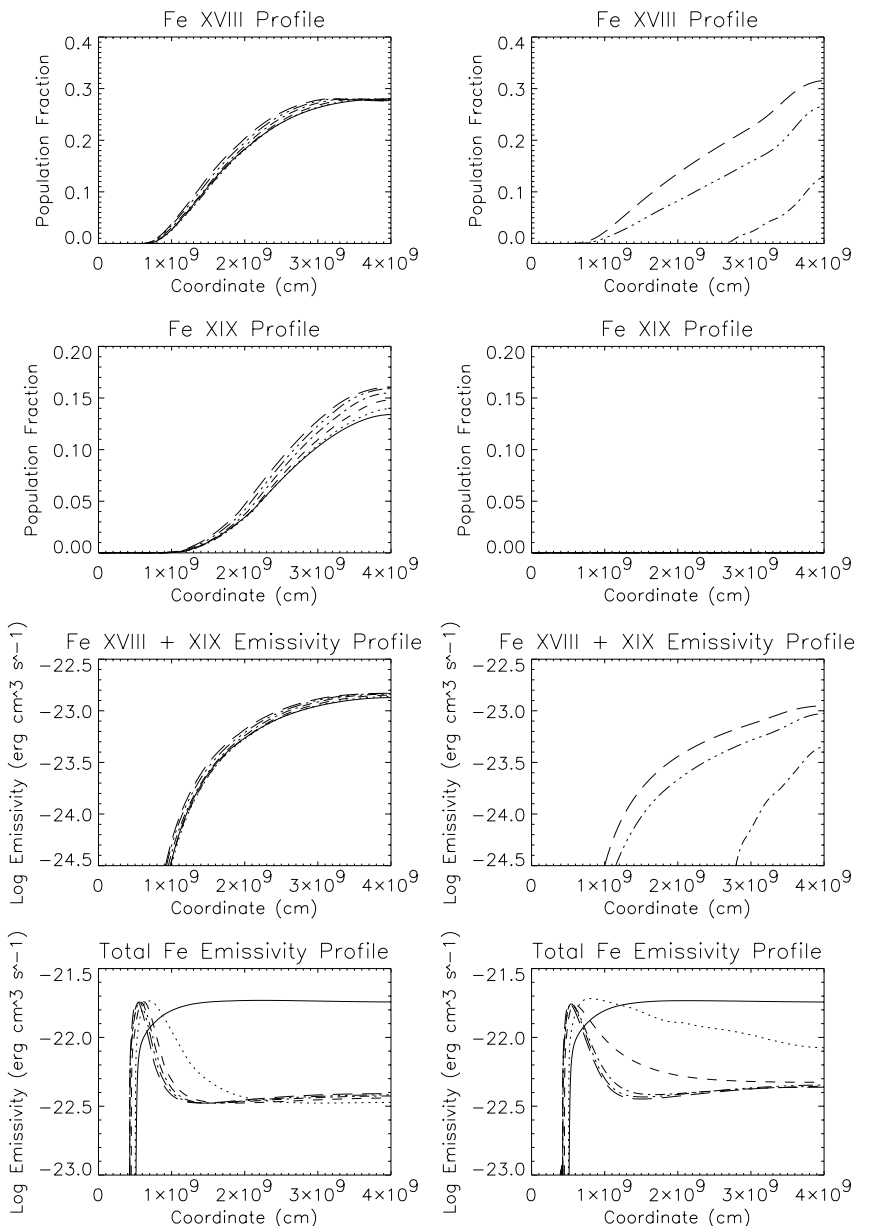

Fig. 10. The top six panels show time series of the Fe XVIII and Fe XIX population fractions and emissivities at: $160 \mathrm{~s}$ (solid), $170 \mathrm{~s}$ (dotted), $180 \mathrm{~s}$ (dashed), $190 \mathrm{~s}$ (dash-dot), $200 \mathrm{~s}$ (dash-dot-dot-dot) and $210 \mathrm{~s}$ (long-dashed). The bottom two panels show time series of the total iron emissivity at: $0 \mathrm{~s}$ (solid), $60 \mathrm{~s}$ (dotted), $90 \mathrm{~s}$ (dashed), $120 \mathrm{~s}$ (dash-dot), $150 \mathrm{~s}$ (dash-dot-dot-dot) and $210 \mathrm{~s}$ (long-dashed). The left-hand panels show the results for simulation $\mathrm{A}$ and the righthand panels show the results for simulation $\mathrm{B}$.
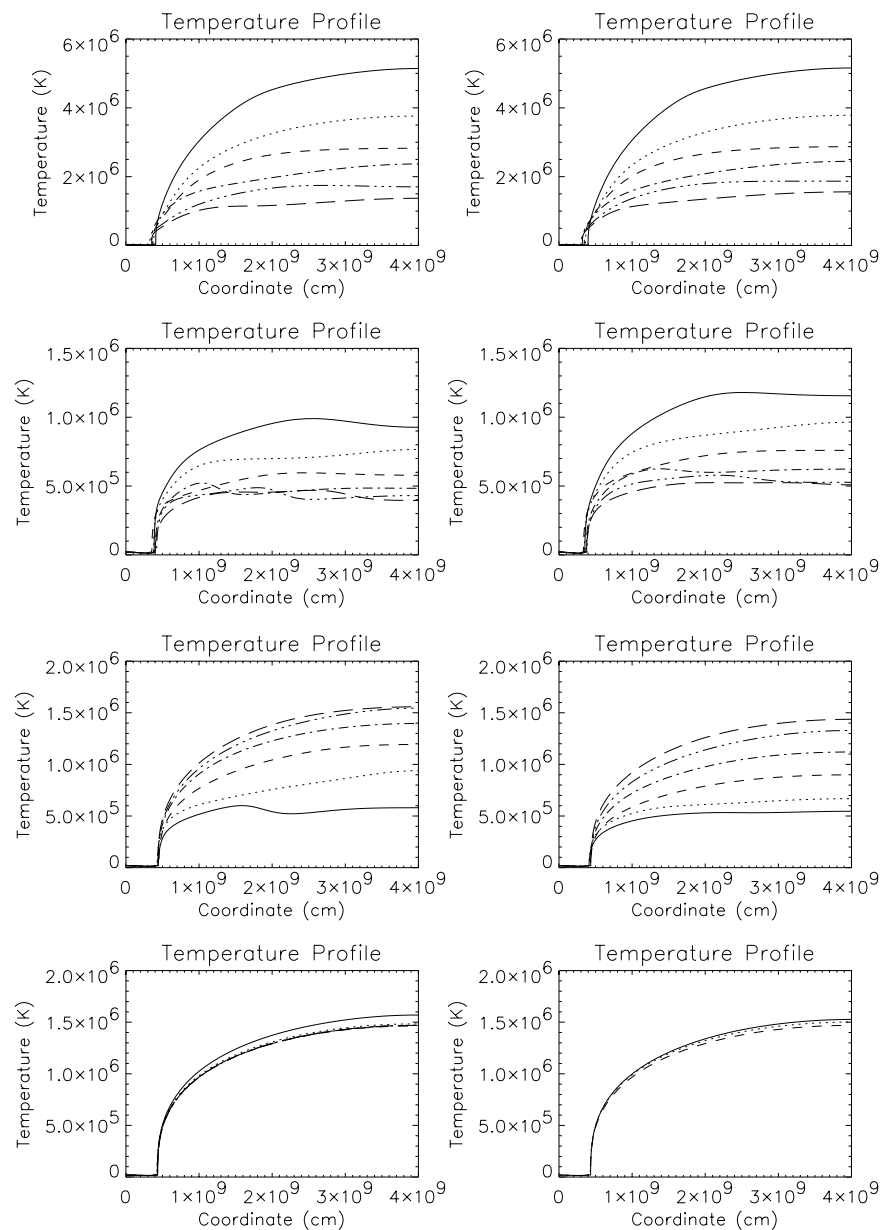

Fig. 11. Time series of the temperature profile for the left-hand region of the loop. Row 1: $300 \mathrm{~s}$ (solid), $400 \mathrm{~s}$ (dotted), $500 \mathrm{~s}$ (dashed), $600 \mathrm{~s}$ (dash-dot), $700 \mathrm{~s}$ (dash-dot-dot-dot) and $800 \mathrm{~s}$ (long-dashed). Row 2: $900 \mathrm{~s}$ (solid), $1000 \mathrm{~s}$ (dotted), $1100 \mathrm{~s}$ (dashed), $1200 \mathrm{~s}$ (dash-dot), $1300 \mathrm{~s}$ (dash-dot-dot-dot) and $1400 \mathrm{~s}$ (long-dashed). Row 3: $1600 \mathrm{~s}$ (solid), $1800 \mathrm{~s}$ (dotted), $2000 \mathrm{~s}$ (dashed), $2200 \mathrm{~s}$ (dash-dot), $2400 \mathrm{~s}$ (dash-dot-dot-dot) and $2600 \mathrm{~s}$ (long-dashed). Row 4: $3000 \mathrm{~s}$ (solid), $4000 \mathrm{~s}$ (dotted), $5000 \mathrm{~s}$ (dashed), $6000 \mathrm{~s}$ (dash-dot), $7000 \mathrm{~s}$ (dashdot-dot-dot) and $8000 \mathrm{~s}$ (long-dashed). The left-hand panels show the results for simulation $\mathrm{A}$ and the right-hand panels show the results for simulation B. 
S. J. Bradshaw et al.: Compact flare emission, Online Material p 5
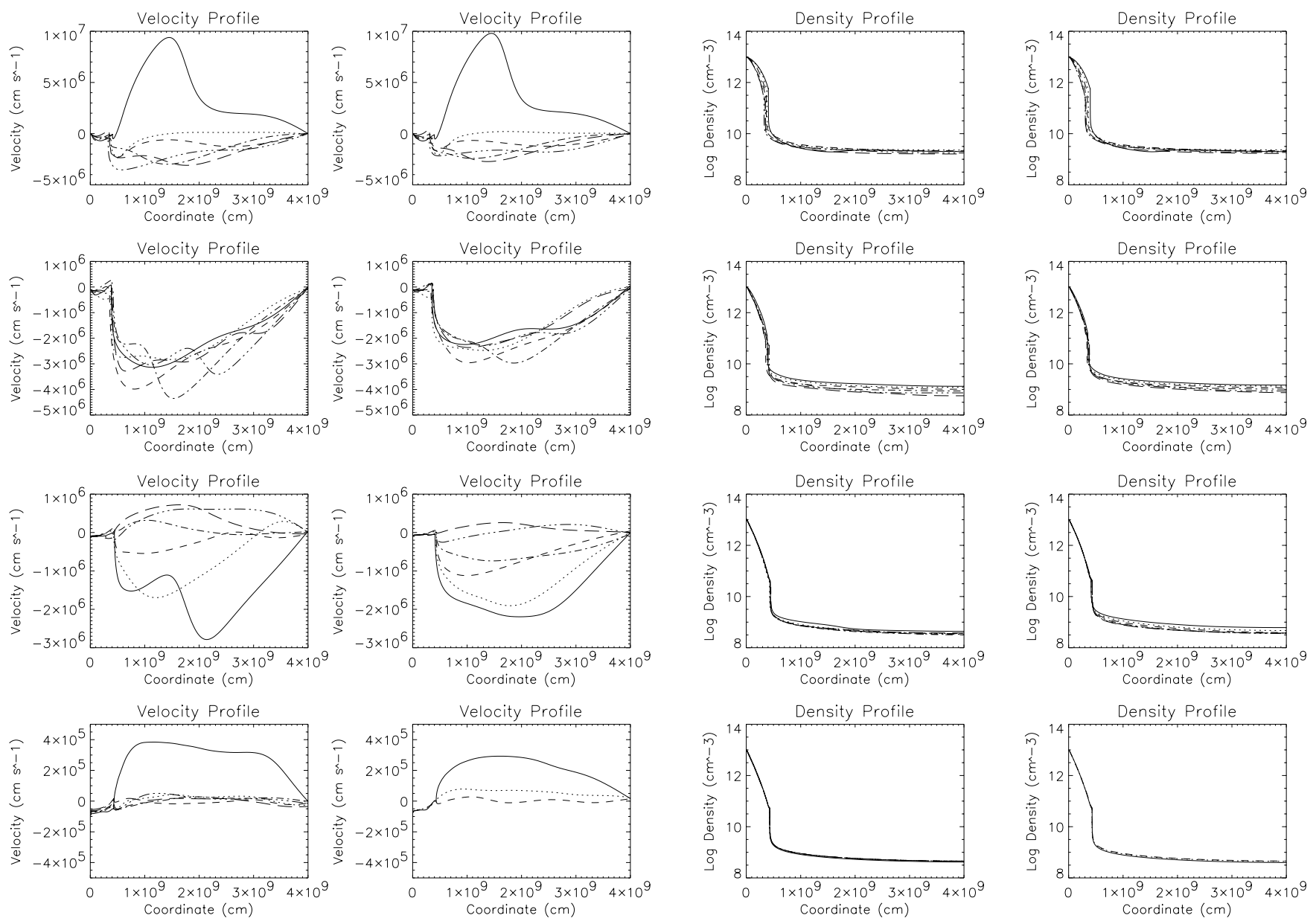

Fig. 12. Time series of the velocity profile for the left-hand region of the loop at the same times as in Fig. 11.
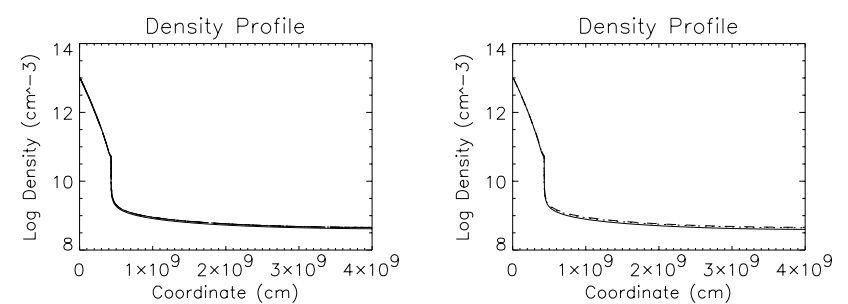

Fig. 13. Time series of the density profile for the left-hand region of the loop at the same times as in Fig. 11. 
S. J. Bradshaw et al.: Compact flare emission, Online Material p 6
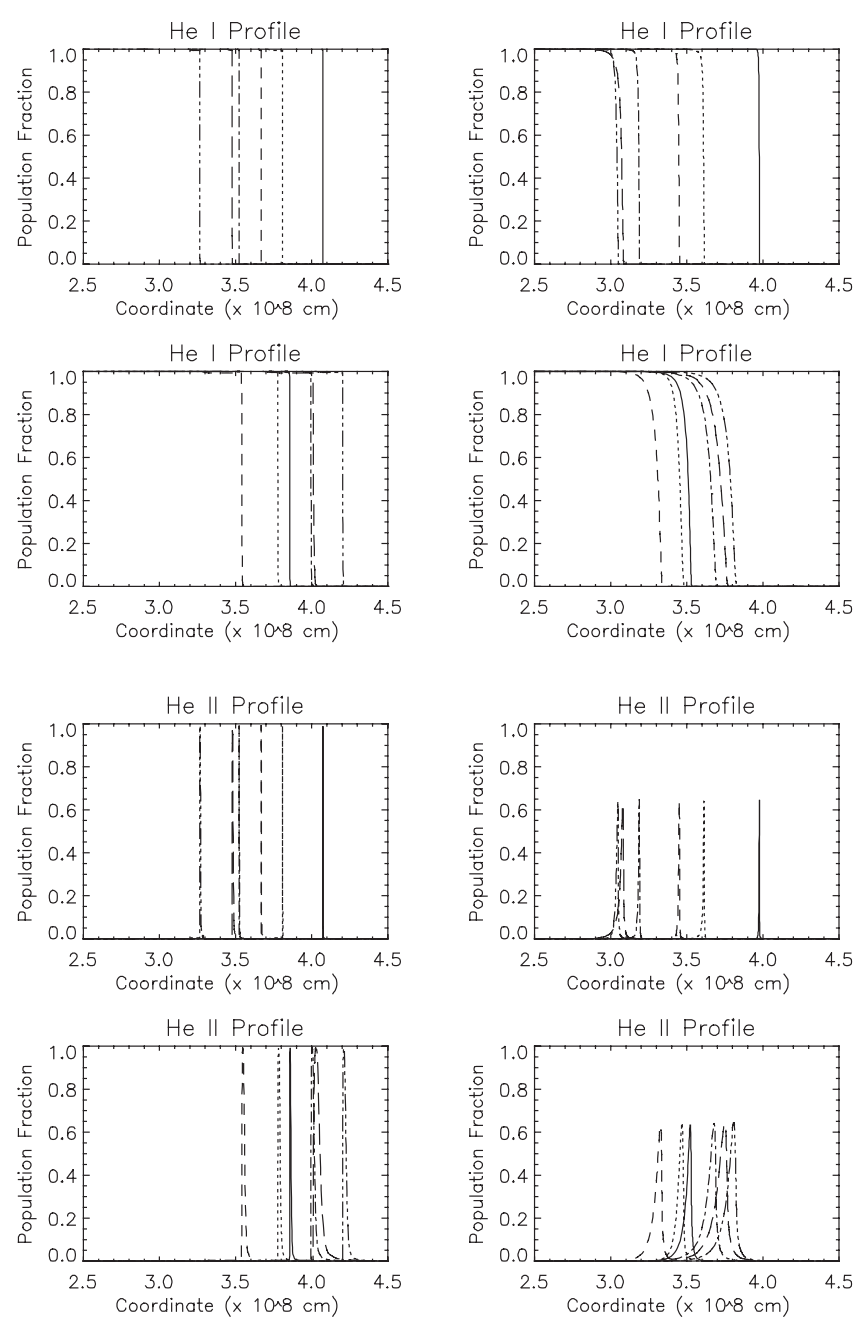
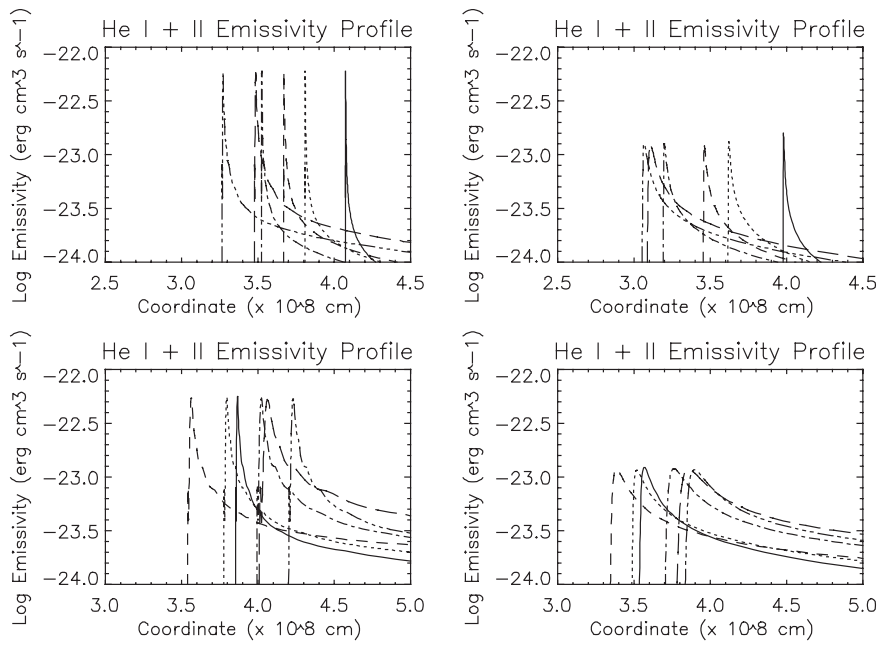

Fig. 15. Time series of the total He I and He II emissivity. Row 1: $300 \mathrm{~s}$ (solid), $400 \mathrm{~s}$ (dotted), $500 \mathrm{~s}$ (dashed), $600 \mathrm{~s}$ (dash-dot), $700 \mathrm{~s}$ (dashdot-dot-dot) and $800 \mathrm{~s}$ (long-dashed). Row 2: $900 \mathrm{~s}$ (solid), $1000 \mathrm{~s}$ (dotted), $1100 \mathrm{~s}$ (dashed), $1200 \mathrm{~s}$ (dash-dot), $1300 \mathrm{~s}$ (dash-dot-dotdot) and $1400 \mathrm{~s}$ (long-dashed). The left-hand panels show the results for simulation A and the right-hand panels show the results for simulation B.

Fig. 14. Time series of the He I and He II population fractions. The first row of plots for each ion shows the population fractions at: $300 \mathrm{~s}$ (solid), $400 \mathrm{~s}$ (dotted), $500 \mathrm{~s}$ (dashed), $600 \mathrm{~s}$ (dash-dot), $700 \mathrm{~s}$ (dashdot-dot-dot) and $800 \mathrm{~s}$ (long-dashed). The second row of plots for each ion shows the population fractions at: $900 \mathrm{~s}$ (solid), $1000 \mathrm{~s}$ (dotted), $1100 \mathrm{~s}$ (dashed), $1200 \mathrm{~s}$ (dash-dot), $1300 \mathrm{~s}$ (dash-dot-dot-dot) and $1400 \mathrm{~s}$ (long-dashed). The left-hand panels show the results for simulation $\mathrm{A}$ and the right-hand panels show the results for simulation B. 
S. J. Bradshaw et al.: Compact flare emission, Online Material $p 7$
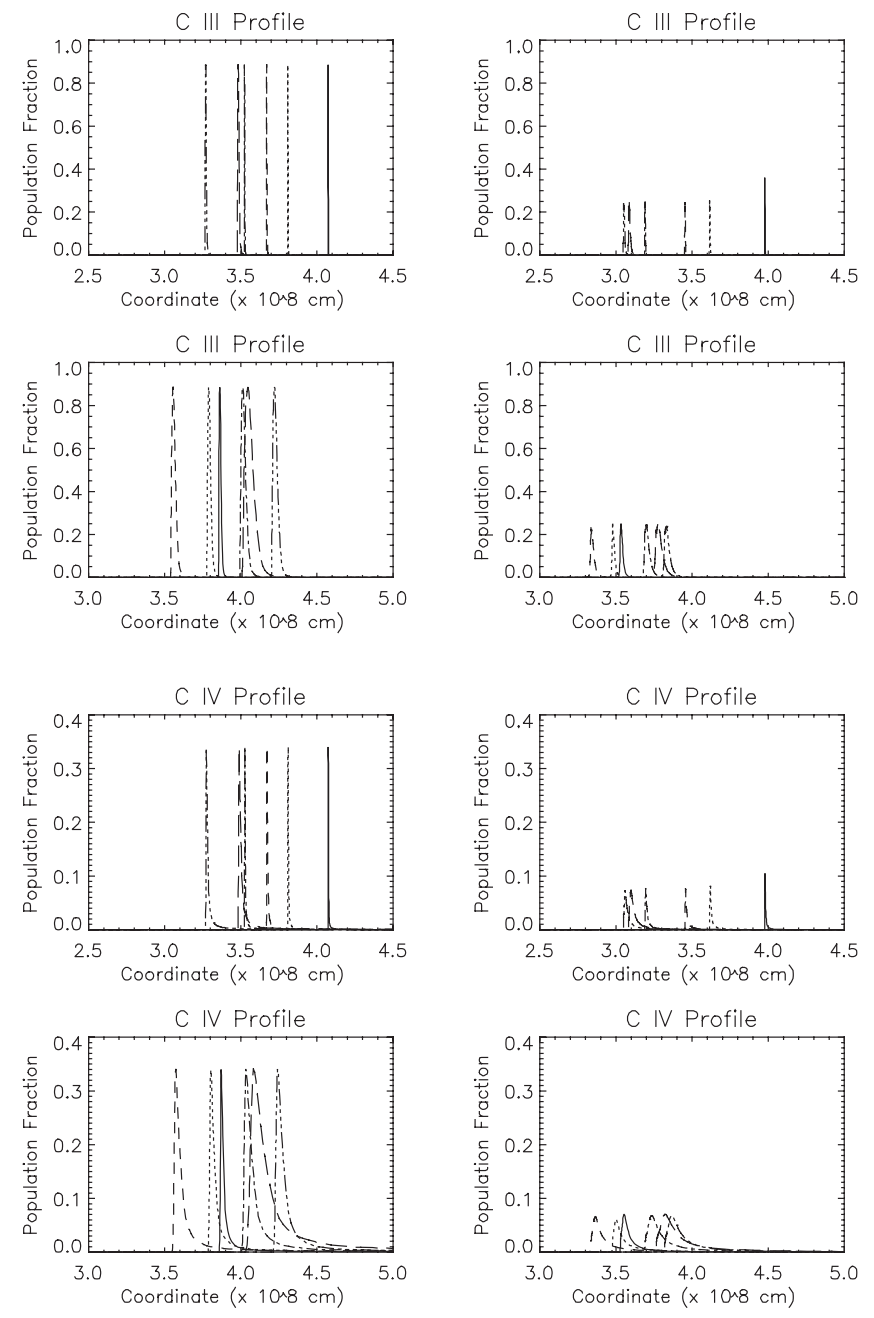

Fig. 16. Time series of the C III and C IV population fractions. The first row of plots for each ion shows the population fractions at: $300 \mathrm{~s}$ (solid), $400 \mathrm{~s}$ (dotted), $500 \mathrm{~s}$ (dashed), $600 \mathrm{~s}$ (dash-dot), $700 \mathrm{~s}$ (dashdot-dot-dot) and $800 \mathrm{~s}$ (long-dashed). The second row of plots for each ion shows the population fractions at: $900 \mathrm{~s}$ (solid), $1000 \mathrm{~s}$ (dotted), $1100 \mathrm{~s}$ (dashed), $1200 \mathrm{~s}$ (dash-dot), $1300 \mathrm{~s}$ (dash-dot-dot-dot) and $1400 \mathrm{~s}$ (long-dashed). The left-hand panels show the results for simulation A and the right-hand panels show the results for simulation B.
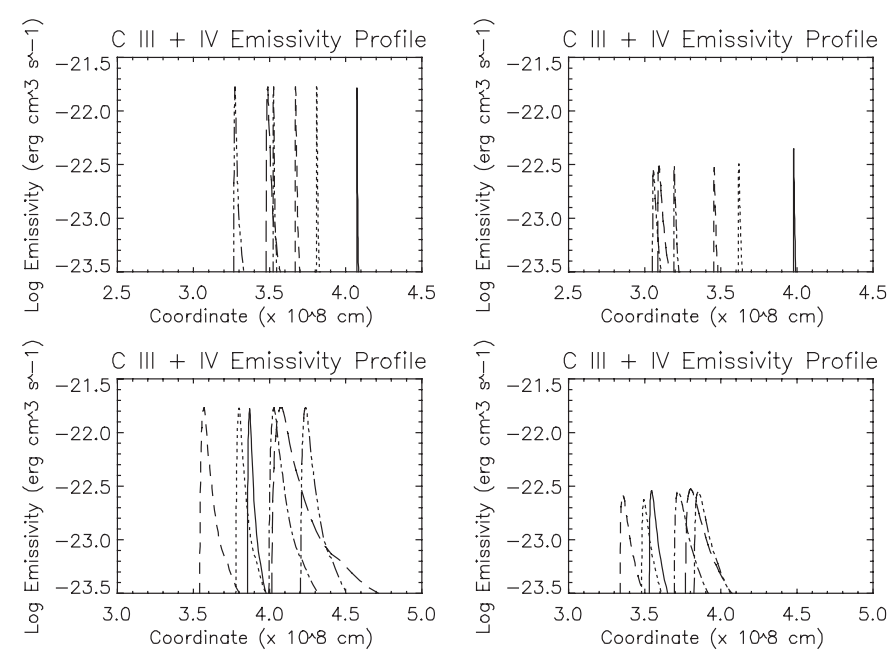

Fig. 17. Time series of the total C III and C IV emissivity. Row 1: $300 \mathrm{~s}$ (solid), $400 \mathrm{~s}$ (dotted), $500 \mathrm{~s}$ (dashed), $600 \mathrm{~s}$ (dash-dot), $700 \mathrm{~s}$ (dashdot-dot-dot) and $800 \mathrm{~s}$ (long-dashed). Row 2: $900 \mathrm{~s}$ (solid), $1000 \mathrm{~s}$ (dotted), $1100 \mathrm{~s}$ (dashed), $1200 \mathrm{~s}$ (dash-dot), $1300 \mathrm{~s}$ (dash-dot-dotdot) and $1400 \mathrm{~s}$ (long-dashed). The left-hand panels show the results for simulation $\mathrm{A}$ and the right-hand panels show the results for simulation B.
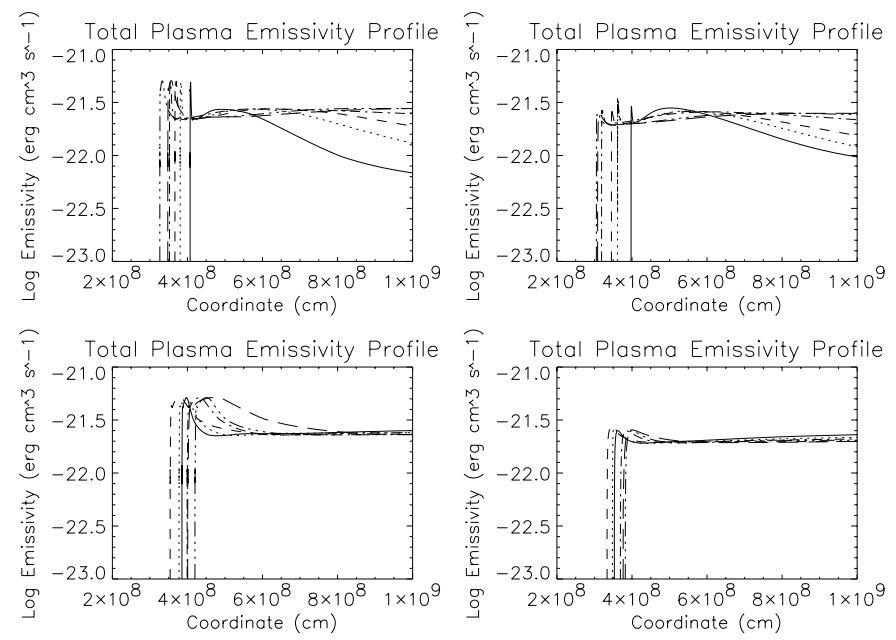

Fig. 18. Time series of the total emissivity in the left-hand footpoint region of the loop. Row 1: $300 \mathrm{~s}$ (solid), $400 \mathrm{~s}$ (dotted), $500 \mathrm{~s}$ (dashed), $600 \mathrm{~s}$ (dash-dot), $700 \mathrm{~s}$ (dash-dot-dot-dot) and $800 \mathrm{~s}$ (long-dashed). Row 2: $900 \mathrm{~s}$ (solid), $1000 \mathrm{~s}$ (dotted), $1100 \mathrm{~s}$ (dashed), $1200 \mathrm{~s}$ (dashdot), $1300 \mathrm{~s}$ (dash-dot-dot-dot) and $1400 \mathrm{~s}$ (long-dashed). The lefthand panels show the results for simulation A and the right-hand panels show the results for simulation B. 
S. J. Bradshaw et al.: Compact flare emission, Online Material $p 8$
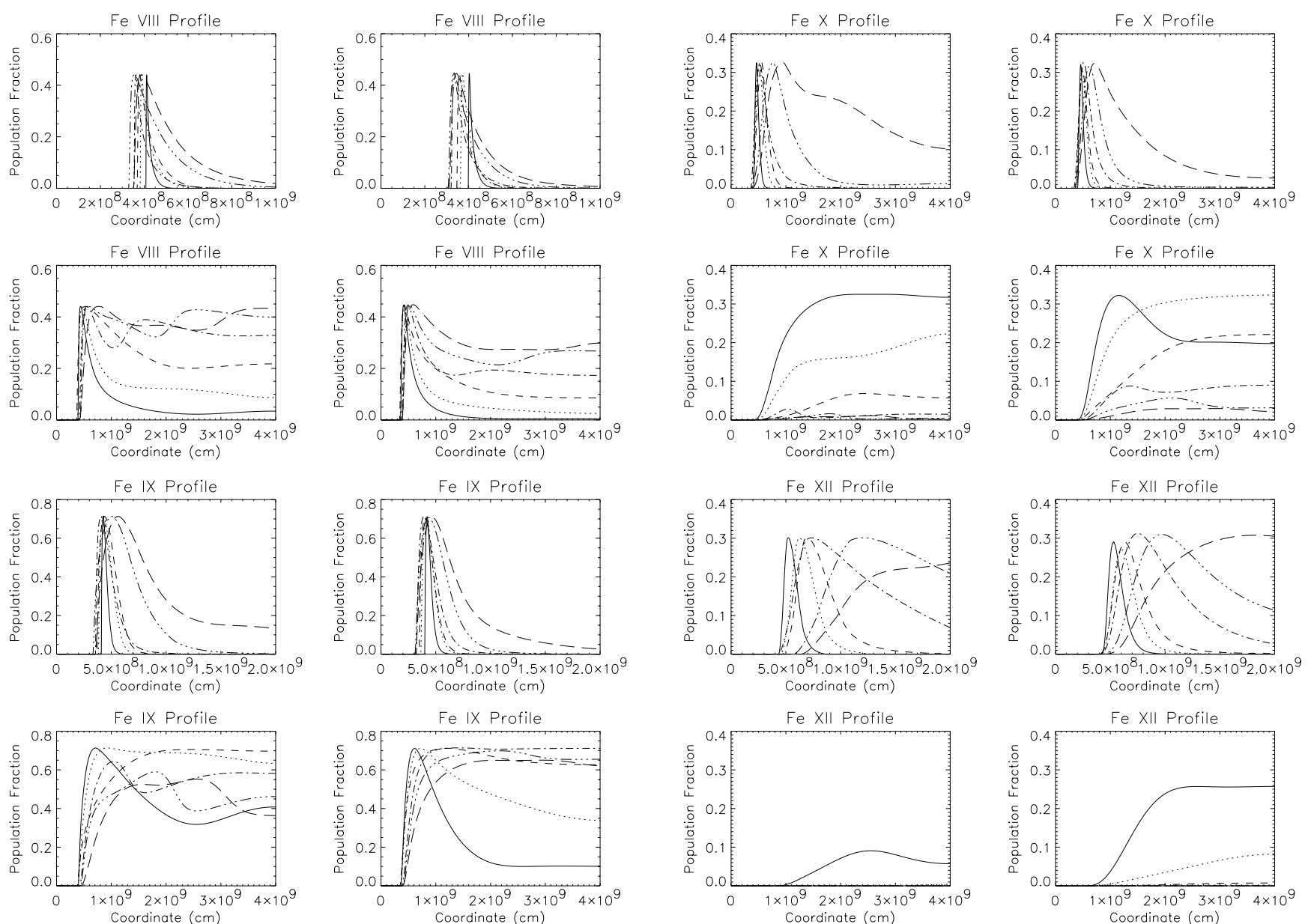

Fig. 19. Time series of the Fe VIII and Fe IX population fractions. The first row of plots for each ion shows the population fractions at: $300 \mathrm{~s}$ (solid), $400 \mathrm{~s}$ (dotted), $500 \mathrm{~s}$ (dashed), $600 \mathrm{~s}$ (dash-dot), $700 \mathrm{~s}$ (dash-dot-dot-dot) and $800 \mathrm{~s}$ (long-dashed). The second row of plots for each ion shows the population fractions at: $900 \mathrm{~s}$ (solid), $1000 \mathrm{~s}$ (dotted), $1100 \mathrm{~s}$ (dashed), $1200 \mathrm{~s}$ (dash-dot), $1300 \mathrm{~s}$ (dash-dot-dotdot) and $1400 \mathrm{~s}$ (long-dashed). The left-hand panels show the results for simulation $\mathrm{A}$ and the right-hand panels show the results for simulation B.
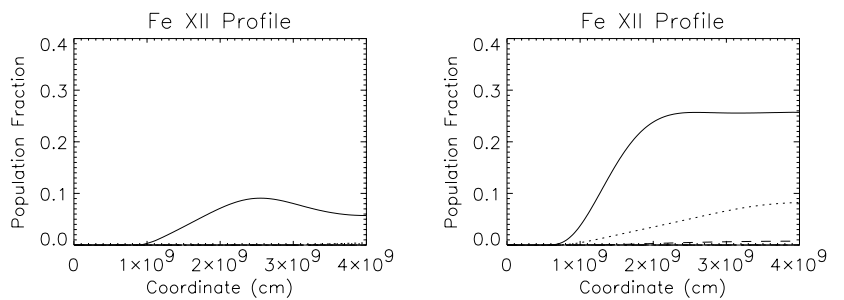

Fig. 20. Time series of the Fe $\mathrm{X}$ and Fe XII population fractions. The first row of plots for each ion shows the population fractions at: $300 \mathrm{~s}$ (solid), $400 \mathrm{~s}$ (dotted), $500 \mathrm{~s}$ (dashed), $600 \mathrm{~s}$ (dash-dot), $700 \mathrm{~s}$ (dashdot-dot-dot) and $800 \mathrm{~s}$ (long-dashed). The second row of plots for each ion shows the population fractions at: $900 \mathrm{~s}$ (solid), $1000 \mathrm{~s}$ (dotted), $1100 \mathrm{~s}$ (dashed), $1200 \mathrm{~s}$ (dash-dot), $1300 \mathrm{~s}$ (dash-dot-dot-dot) and $1400 \mathrm{~s}$ (long-dashed). The left-hand panels show the results for simulation $\mathrm{A}$ and the right-hand panels show the results for simulation B. 

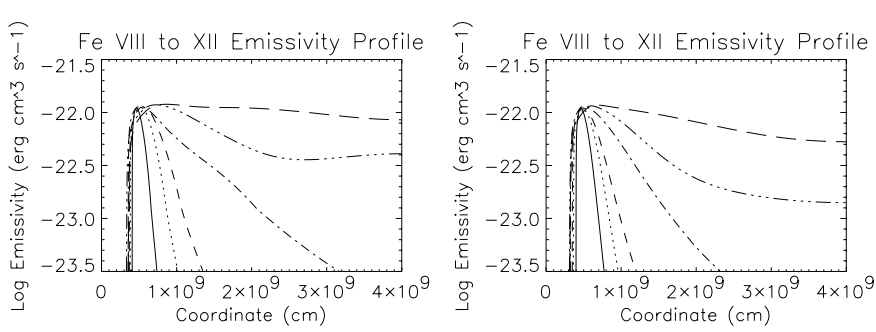

I Fe VIII to XII Emissivity Profile
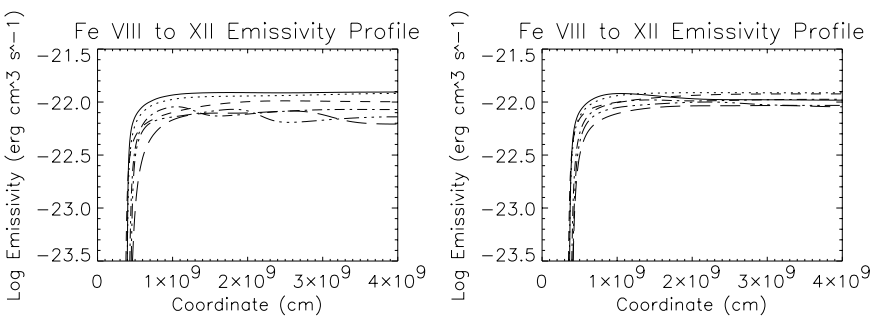

Fig. 21. Time series of the total Fe VIII to Fe XII emissivity. Row 1: $300 \mathrm{~s}$ (solid), $400 \mathrm{~s}$ (dotted), $500 \mathrm{~s}$ (dashed), $600 \mathrm{~s}$ (dash-dot), $700 \mathrm{~s}$ (dash-dot-dot-dot) and $800 \mathrm{~s}$ (long-dashed). Row 2: $900 \mathrm{~s}$ (solid), $1000 \mathrm{~s}$ (dotted), $1100 \mathrm{~s}$ (dashed), $1200 \mathrm{~s}$ (dash-dot), $1300 \mathrm{~s}$ (dashdot-dot-dot) and $1400 \mathrm{~s}$ (long-dashed). The left-hand panels show the results for simulation A and the right-hand panels show the results for simulation B.
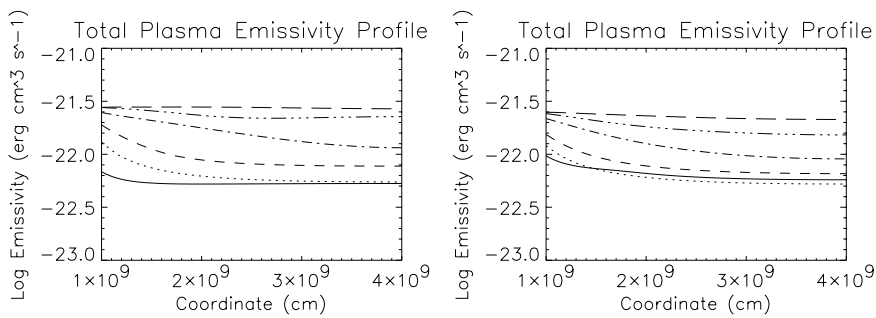

Total Plasma Emissivity Profile

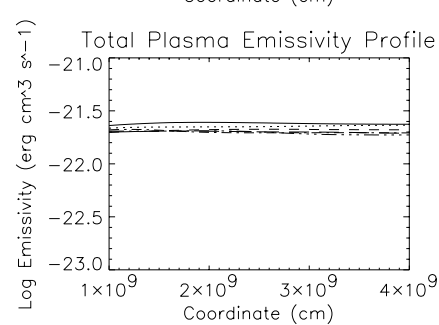

Fig. 22. Time series of the total emissivity in the left-hand coronal region of the loop. Row 1: $300 \mathrm{~s}$ (solid), $400 \mathrm{~s}$ (dotted), $500 \mathrm{~s}$ (dashed), $600 \mathrm{~s}$ (dash-dot), $700 \mathrm{~s}$ (dash-dot-dot-dot) and $800 \mathrm{~s}$ (long-dashed). Row 2: $900 \mathrm{~s}$ (solid), $1000 \mathrm{~s}$ (dotted), $1100 \mathrm{~s}$ (dashed), $1200 \mathrm{~s}$ (dashdot), $1300 \mathrm{~s}$ (dash-dot-dot-dot) and $1400 \mathrm{~s}$ (long-dashed). The lefthand panels show the results for simulation A and the right-hand panels show the results for simulation B.
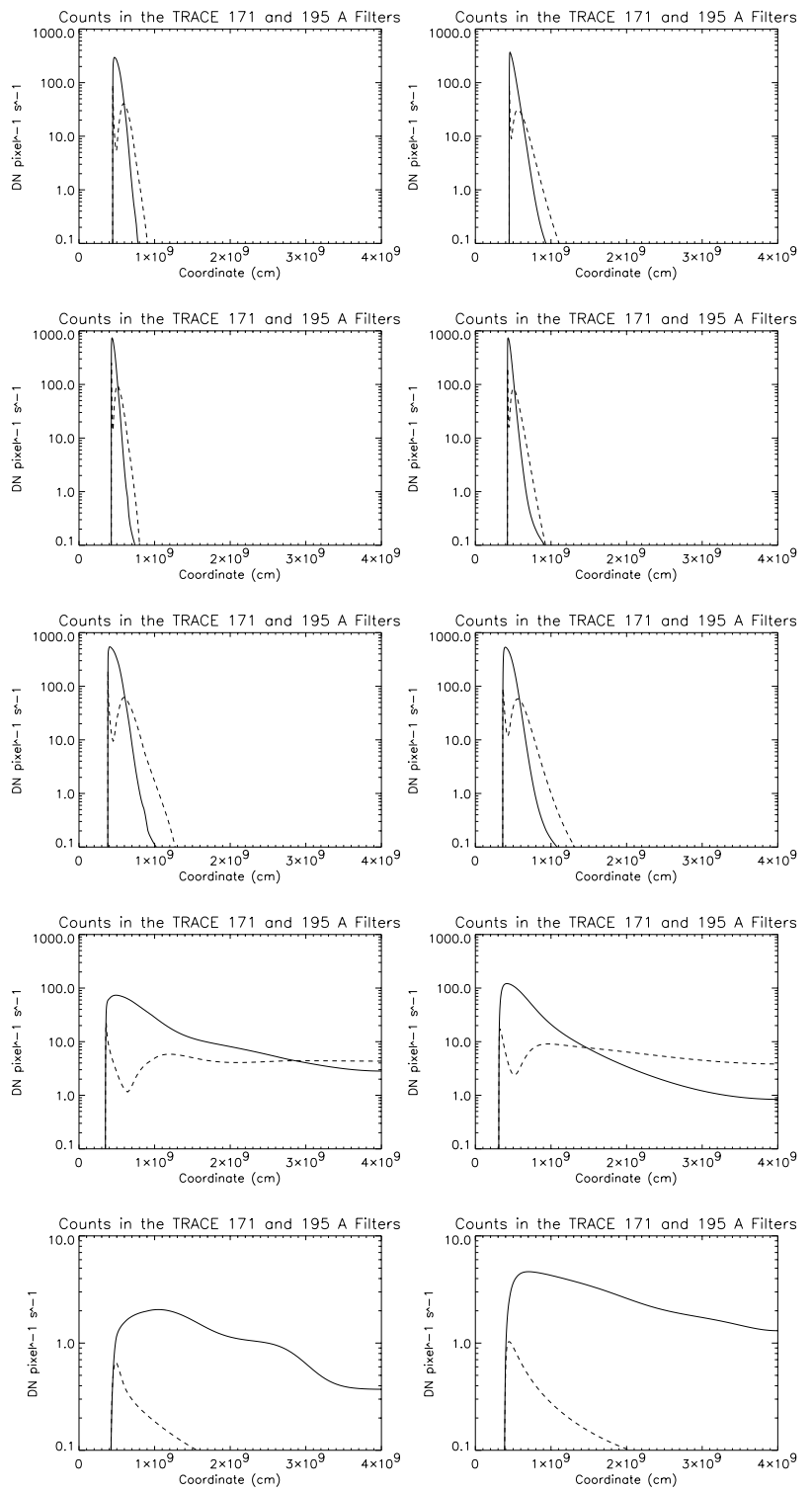

Fig. 23. Profiles of counts in the TRACE $171 \AA$ (solid) and $195 \AA$ (dashed) filters for simulation A (left-hand column) and simulation B (right-hand column). Row 1: 90 s. Row 2: 210 s. Row 3: 400 s. Row 4: 800 s. Row 5: 1400 s. 
S. J. Bradshaw et al.: Compact flare emission, Online Material p 10
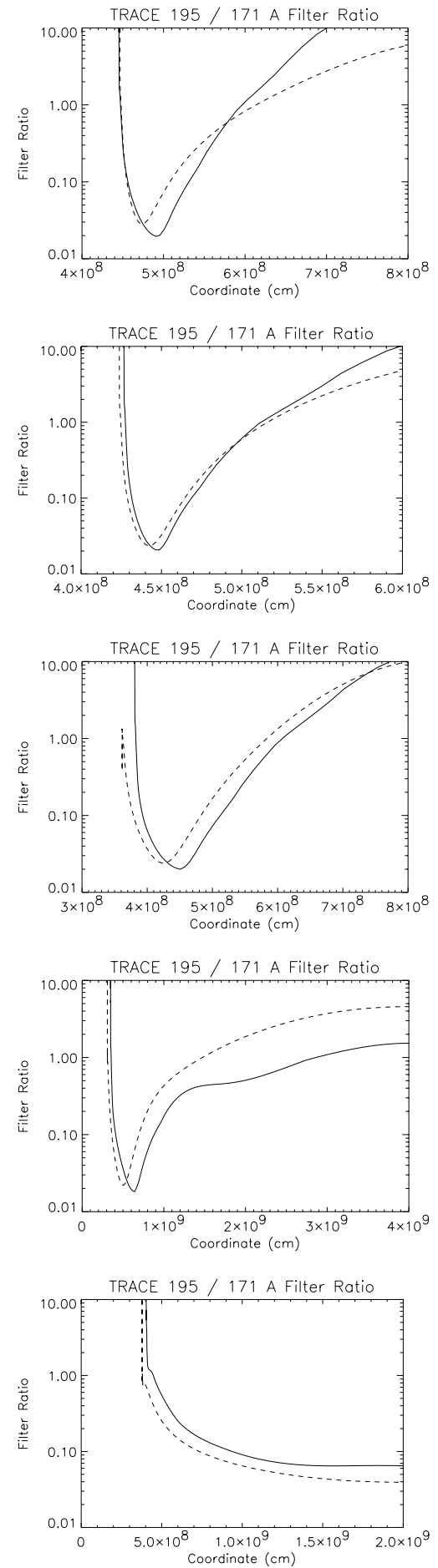

Fig. 24. Profiles of the filter ratio for simulation A (solid) and simulation B (dashed). Top-left: $90 \mathrm{~s}$. Top-right: $210 \mathrm{~s}$. Middle-left: $400 \mathrm{~s}$. Middle-right: 800 s. Bottom: 1400 s. 